\title{
Classification of Singular Fibres on Rational Elliptic Surfaces in Characteristic Three
}

\author{
Tyler Jarvis, William E. Lang, \\ Nansen Petrosyan, Gretchen Rimmasch, \\ Julie Rogers, Erin D. Summers
}

October 19, 2018

\section{Introduction}

\subsection{Background and Overview}

In $[7$ U. Persson classified all possible configurations of singular fibres on rational elliptic surfaces over the complex numbers. He found 279 different configurations. R. Miranda 4] used a more combinatorial method to redo Persson's list. W. Lang 2] continued the classification for characteristic two. Following the method of Lang, we list all possible configurations of singular fibres on rational elliptic surfaces in characteristic three. In total, we find that 267 distinct configurations exist.

Let $k$ be an algebraically closed field of characteristic three. Let $f: X \rightarrow \mathbb{P}^{1}$ be a rational elliptic surface over $k$. We will assume that the surface is relatively minimal and that $f$ has a section. Such a surface has an expression in terms of a Weierstrass equation

$$
y^{2}=x^{3}+b_{2} x^{2}-b_{4} x+b_{6},
$$

with $b_{2}, b_{4}, b_{6} \in k[t]$ and degree $b_{i} \leq i$. The discriminant $\Delta$ for this surface is

$$
\Delta=-b_{2}^{2}\left(b_{2} b_{6}-b_{4}^{2}\right)+b_{4}^{3} .
$$

Assuming that the surface has good reduction at infinity, the discriminant $\Delta$ of the surface is a polynomial in $t$ of degree exactly 12. According to Tate's algorithm [9], the surface has a singular fibre of multiplicative type at a given point $t=p$ of the base if and only if $\Delta(p)=0$ but $b_{2}(p) \neq 0$, and a singular fibre of additive type at $t=p$ if and only if $\Delta(p)=0$ and $b_{2}(p)=0$. There are four general configurations of singular fibres that we will consider:

1. $\quad b_{2} \neq 0$ and all the singular fibres are multiplicative.

2. $\quad b_{2} \neq 0$ and one of the singularities is additive, and any remaining singularities are multiplicative. 
3. $\quad b_{2} \neq 0$ and two of the singularities are additive, and any remaining singularities are multiplicative.

4. $\quad b_{2} \equiv 0$, in which case all the singularities are additive, and there can be more than two distinct additive singularities. In this case the $j$-invariant is also identically zero.

We will treat the first case separately and the other three together. The structure of the paper will be as follows. First, some preliminaries relating to all surfaces will be discussed. We will then consider those surfaces which have only multiplicative type singular fibres, followed by those surfaces with at least one additive type singular fibre. Finally we will give a summary of the results.

\subsection{Acknowledgments}

We are grateful to Heidi Jarvis for her expert help with proofreading and typesetting. Research of the first author was supported in part by NSF grant DMS0105788. The last four authors were supported in part by graduate research assistantships from the Brigham Young University mathematics department.

\section{Preliminaries}

Let $f: X \rightarrow \mathbb{P}^{1}$ be a rational elliptic surface over an algebraically closed field $k$ of characteristic three. We assign several invariants to each singular fibre, as given in Table 1 First we list the Kodaira type F. But, unlike in characteristic zero, each singularity may appear in different ways, for example with different orders of vanishing of $\Delta$, so we also list Lang's case number $\mathrm{L}$ from the classification in [3] of isolated additive type singular fibres on a rational elliptic surface in characteristic three. Lang's normal forms from that classification play a key role in this project.

The order of vanishing of $\Delta$ is denoted by $\delta$. Associated with each singular fibre is a lattice $\Lambda$ of rank $r$, generated by the irreducible components of the singular fibre which do not meet the zero section. The lattice $\Lambda$ is a sublattice of the Nèron-Severi lattice of the entire surface, with discriminant $d$, and is orthogonal to the lattices associate to the other fibres.

Table 1: Fibre Invariants

\begin{tabular}{|c|c|c|c|c|c|}
\hline $\mathbf{F}$ & $\mathbf{L}$ & $\boldsymbol{\delta}$ & $\mathbf{r}$ & $\boldsymbol{\Lambda}$ & $\mathbf{d}$ \\
\hline \hline $\mathrm{I}_{\mathbf{n}}$ & & $\mathrm{n}$ & $\mathrm{n}-1$ & $\mathrm{~A}_{\boldsymbol{n}-1}$ & $\mathrm{n}$ \\
\hline $\mathrm{II}$ & $1 \mathrm{~A}$ & 3 & 0 & $\{0\}$ & 0 \\
\hline II & $1 \mathrm{~B}$ & 4 & 0 & $\{0\}$ & 0 \\
\hline II & $1 \mathrm{C}$ & 6 & 0 & $\{0\}$ & 0 \\
\hline II & $1 \mathrm{D}$ & 7 & 0 & $\{0\}$ & 0 \\
\hline II & $1 \mathrm{E}$ & 9 & 0 & $\{0\}$ & 0 \\
\hline
\end{tabular}

continued on next page 


continued from previous page
\begin{tabular}{|c|c|c|c|c|c|}
\hline F & $\mathbf{L}$ & $\boldsymbol{\delta}$ & $\mathbf{r}$ & $\boldsymbol{\Lambda}$ & $\mathbf{d}$ \\
\hline \hline $\mathrm{II}$ & $1 \mathrm{~F}$ & 12 & 0 & $\{0\}$ & 0 \\
\hline $\mathrm{III}$ & 2 & 3 & 1 & $\mathrm{~A}_{1}$ & 2 \\
\hline $\mathrm{IV}$ & $3 \mathrm{~A}$ & 5 & 2 & $\mathrm{~A}_{2}$ & 3 \\
\hline $\mathrm{IV}$ & $3 \mathrm{~B}$ & 6 & 2 & $\mathrm{~A}_{2}$ & 3 \\
\hline $\mathrm{IV}$ & $3 \mathrm{C}$ & 8 & 2 & $\mathrm{~A}_{2}$ & 3 \\
\hline $\mathrm{IV}$ & $3 \mathrm{D}$ & 9 & 2 & $\mathrm{~A}_{2}$ & 3 \\
\hline $\mathrm{IV}$ & $3 \mathrm{E}$ & 12 & 2 & $\mathrm{~A}_{2}$ & 3 \\
\hline $\mathrm{I}_{0}^{*}$ & $4 \mathrm{~A}$ & 6 & 4 & $\mathrm{D}_{4}$ & 4 \\
\hline $\mathrm{I}_{0}^{*}$ & $4 \mathrm{~B}$ & 6 & 4 & $\mathrm{D}_{4}$ & 4 \\
\hline $\mathrm{I}_{1}^{*}$ & $5 \mathrm{~A}$ & 7 & 5 & $\mathrm{D}_{5}$ & 4 \\
\hline $\mathrm{I}_{2}^{*}$ & $5 \mathrm{~B}$ & 8 & 6 & $\mathrm{D}_{6}$ & 4 \\
\hline $\mathrm{I}_{3}^{*}$ & $5 \mathrm{C}$ & 9 & 7 & $\mathrm{D}_{7}$ & 4 \\
\hline $\mathrm{I}_{4}^{*}$ & $5 \mathrm{D}$ & 10 & 8 & $\mathrm{D}_{8}$ & 4 \\
\hline $\mathrm{IV}^{*}$ & $6 \mathrm{~A}$ & 9 & 6 & $\mathrm{E}_{6}$ & 3 \\
\hline $\mathrm{IV}^{*}$ & $6 \mathrm{~B}$ & 10 & 6 & $\mathrm{E}_{6}$ & 3 \\
\hline $\mathrm{IV}^{*}$ & $6 \mathrm{C}$ & 12 & 6 & $\mathrm{E}_{6}$ & 3 \\
\hline III $^{*}$ & 7 & 9 & 7 & $\mathrm{E}_{7}$ & 2 \\
\hline $\mathrm{II}^{*}$ & $8 \mathrm{~A}$ & 11 & 8 & $\mathrm{E}_{8}$ & 1 \\
\hline $\mathrm{II}^{*}$ & $8 \mathrm{~B}$ & 12 & 8 & $\mathrm{E}_{8}$ & 1 \\
\hline
\end{tabular}

These invariants exclude certain configurations of singular fibres, as they must satisfy the conditions given in the following three lemmas.

Lemma $\mathbf{A}([4])$. On a rational elliptic surface, $\sum \delta_{\mathrm{F}}=12$ and $\sum \mathrm{r}_{\mathrm{F}} \leq 8$.

Lemma $\mathrm{B}([\mathbf{5}])$. If $\sum \mathrm{r}_{\mathrm{F}}=8$, then $\prod \mathrm{d}_{\mathrm{F}}$ is a perfect square.

Lemma $\mathbf{C}([\mathbf{8}])$. In order for a given configuration of singular fibres to exist, the associated lattice $\oplus_{\mathrm{F}} \Lambda_{\mathrm{F}}$ must embed in the root lattice $\mathrm{E}_{8}$.

Oguiso and Shioda [6] provide a list, originally due to Dynkin [1], of lattices which embed in the root lattice $\mathrm{E}_{8}$. For the convenience of the reader we include the complement of that list here; namely, we list all direct sums of lattices of type $A_{n}, D_{n}$ or $E_{n}$ of rank no more than eight which do not embed in $E_{8}$. Note that if the rank of such a lattice is 6 or less, it always embeds in $E_{8}$.

Table 2: Lattices which are direct sums of $A_{n}$ 's, $D_{n}$ 's,or $E_{n}$ 's, which do not embed in $E_{8}$.

\begin{tabular}{|l|l|}
\hline rank 7 & rank 8 \\
\hline$A_{2} \oplus A_{1}^{\oplus 5}$ & $A_{2} \oplus A_{1}^{\oplus 6}$ \\
& $A_{2}^{\oplus 2} \oplus A_{1}^{\oplus 4}$ \\
& $A_{3} \oplus A_{1}^{\oplus 5}$ \\
\hline \multicolumn{2}{|r|}{ continued on next page }
\end{tabular}




continued from previous page
\begin{tabular}{|l|l|}
\hline rank 7 & rank 8 \\
\hline$A_{2}^{\oplus 2} \oplus A_{1}^{\oplus 3}$ & $A_{3} \oplus A_{2} \oplus A_{1}^{\oplus 3}$ \\
& $A_{2}^{\oplus 3} \oplus A_{1}^{\oplus 2}$ \\
\hline$A_{3} \oplus A_{2}^{\oplus 2}$ & $A_{3} \oplus A_{2}^{\oplus 2} \oplus A_{1}$ \\
& $A_{3}^{\oplus 2} \oplus A_{2}$ \\
& $A_{4} \oplus A_{2}^{\oplus 2}$ \\
& $A_{5} \oplus A_{3}$ \\
& $A_{6} \oplus A_{2}$ \\
\hline$A_{4} \oplus A_{1}^{\oplus 3}$ & $A_{4} \oplus A_{1}^{\oplus 4}$ \\
& $A_{4} \oplus A_{2} \oplus A_{1}^{\oplus 2}$ \\
& $A_{4} \oplus A_{3} \oplus A_{1}$ \\
& $A_{5} \oplus A_{1}^{\oplus 3}$ \\
& $A_{6} \oplus A_{1}^{\oplus 2}$ \\
\hline$D_{4} \oplus A_{2} \oplus A_{1}$ & $D_{4} \oplus A_{2} \oplus A_{1}^{\oplus 2}$ \\
& $D_{4} \oplus A_{2}^{\oplus 2}$ \\
& $D_{4} \oplus A_{3} \oplus A_{1}$ \\
& $D_{5} \oplus A_{2} \oplus A_{1}$ \\
& $D_{6} \oplus A_{2}$ \\
& $D_{7} \oplus A_{1}$ \\
\hline & $D_{5} \oplus A_{1}^{\oplus 3}$ \\
& $E_{6} \oplus A_{1}^{\oplus 2}$ \\
\hline
\end{tabular}

We also note that we have not used the J-map in our classifications at all, although it was used in the classifications done in other charactersitics. We do not use it here because there were only a few cases where the J-map provided simple results, and each of these cases could be ruled out using another lemma which was required for more cases.

\section{Surfaces with Singular Fibres of Multiplica- tive Type}

We now consider the case where all singular fibres are of multiplicative type.

\subsection{General Information}

For convenience, when we write out a configuration of multiplicative fibres, we will write $n_{1} n_{2} n_{3} \ldots$ instead of writing $I_{n_{1}} I_{n_{2}} I_{n_{3}} \ldots$ Repeated terms will be denoted by exponents, so $53^{2} 1$ denotes the configuration $\mathrm{I}_{5} \mathrm{I}_{3} \mathrm{I}_{3} \mathrm{I}_{1}$.

As mentioned before, a rational elliptic surface has a multiplicative fibre over a point $t=p$ precisely if $\Delta$ has a root at $p$ but $b_{2}$ does not. Since $b_{2}$ is 
a non-zero polynomial of degree two, we have two cases: either $b_{2}$ has distinct roots, or $b_{2}$ has a repeated root. In all cases where a given configuration exists, we will give an example with distinct roots of $b_{2}$. It is interesting to note that there are 11 cases which exist when $b_{2}$ has distinct roots but do not exist when $\mathrm{b}_{2}$ has a repeated root; namely $531^{4}, 81^{4}, 52^{2} 1^{3}, 541^{3}, 3^{2} 2^{2} 1^{2}, 62^{2} 1^{2}$, $5321^{2}, 821^{2}, 5^{2} 1^{2}, 432^{2} 1$, and $4^{2} 2^{2}$.

We begin by reducing the discriminant using standard transformations to a normal form, given in equations (3.1.2) and (3.1.4). In the case that $b_{2}$ has distinct roots, we use a fractional linear transformation to move these roots to zero and infinity, and in the case that $b_{2}$ has a double root, we move that unique root to infinity.

Proposition 3.1.1. Consider a minimal rational elliptic surface over $\mathbb{P}_{\mathrm{t}}^{1}$ with Weierstrauss equation

$$
y^{2}=x^{3}+b_{2} x^{2}-b_{4} x+b_{6}
$$

where the $b_{i}$ are polynomials in $t$ of degree no more than $i$. If all of the singular fibres are of multiplicative type, and if the roots of $\mathrm{b}_{2}$ are distinct, then up to automorphism of the surface and the base, the discriminant of the surface is a degree twelve polynomial of the form

$$
\Delta=t^{12}+l t^{10}+t^{3} P_{6}(t)+n t^{2}+m
$$

where $\mathrm{m}^{3}=\mathrm{n}^{2} \mathrm{l}^{3} \neq 0$, and where $\mathrm{P}_{6}(\mathrm{t})$ is a polynomial of degree 6 .

Conversely, given any $\Delta$ of the form (3.1.2) there is a rational elliptic surface with Weierstrass equation (3.1.1) and with roots of $\mathrm{b}_{2}$ at 0 and $\infty$. If such a surface exists with $\Delta$ of form [3.1.2), we may, up to automorphism of the base, assume that one root of $\Delta$ occurs at $\mathrm{t}=1$.

Proof. PGL(2) acts three-transitively on $\mathbb{P}^{1}$, thus we can find a transformation that sends one root of $b_{2}$ to infinity and one root to zero. Therefore, by a standard change of variables, we may assume $b_{2}=t$.

Let

$$
\mathrm{b}_{4}=\xi_{4} \mathrm{t}^{4}+\xi_{3} \mathrm{t}^{3}+\xi_{2} \mathrm{t}^{2}+\xi_{1} \mathrm{t}+\xi_{0},
$$

and

$$
b_{6}=\gamma_{6} t^{6}+\gamma_{5} t^{5}+\gamma_{4} t^{4}+\gamma_{3} t^{3}+\gamma_{2} t^{2}+\gamma_{1} t+\gamma_{0}
$$

where $\xi_{i}, \gamma_{j} \in k$. By substituting $b_{2}, b_{4}, b_{6}$ into the equation of $\Delta$ and simplifying we get

$$
\begin{aligned}
\Delta= & \xi_{4}^{3} t^{12}+\xi_{4}^{2} t^{10}+\left(-\gamma_{6}-\xi_{3} \xi_{4}\right) t^{9}+\left(\xi_{3}^{2}-\gamma_{5}-\xi_{2} \xi_{4}\right) t^{8} \\
& +\left(-\gamma_{4}-\xi_{1} \xi_{4}-\xi_{2} \xi_{3}\right) t^{7}+\left(-\gamma_{3}+\xi_{2}^{3}-\xi_{0} \xi_{4}-\xi_{1} \xi_{3}+\xi_{2}^{2}\right) t^{6} \\
& +\left(-\gamma_{2}-\xi_{0} \xi_{3}-\xi_{1} \xi_{2}\right) t^{5}+\left(-\gamma_{1}-\xi_{1}^{2}-\xi_{0} \xi_{2}^{2}\right) t^{4} \\
& +\left(-\gamma_{0}-\xi_{0} \xi_{1}+\xi_{1}^{3}\right) t^{3}+\xi_{0}^{2} t^{2}+\xi_{0}^{3} .
\end{aligned}
$$

So

$$
\Delta=a^{3} t^{12}+a^{2} t^{10}+t^{3} P_{6}+b^{2} t^{2}+b^{3}
$$


for some $a, b \in k$, and $P_{6} \in k[t]$ is a polynomial of degree 6 in $t$. Since the surface has good reduction at infinity, we have $a \neq 0$; so using the transformation $x=a^{\frac{1}{2}} x^{\prime}$ and $y=a^{\frac{3}{4}} y^{\prime}$, we obtain

$$
\Delta=\left(t^{12}+l t^{10}+t^{3} P_{6}+m t^{2}+n\right),
$$

where

$$
l=a^{-1}, \quad m=b^{2} a^{-3}, \quad n=b^{3} a^{-3},
$$

and hence $\mathrm{m}^{3}=\mathrm{n}^{2} \mathrm{l}^{3}$.

Conversely, for any $l, m, n \in k$ such that $m^{3}=n^{2} l^{3} \neq 0$ and $P_{6} \in k[t]$, we may invert the previous steps; first, by using the standard transformation $x^{\prime}=a^{-\frac{1}{2}} x$ and $y^{\prime}=a^{-\frac{3}{4}} y$ where $a=l^{-1}$, and $b=n m^{-1}$, giving $\Delta=a^{3} t^{12}+$ $a^{2} t^{10}+t^{3} P_{6}+b^{2} t^{2}+b^{3}$. Letting

$$
\xi_{4}=a, \quad \xi_{0}=b, \quad \epsilon_{0}=\epsilon_{2}=0, \quad \epsilon_{1}=1
$$

and $P_{6}(t)=\sum_{i=0}^{6} \psi_{i} t^{i}$, with $\psi_{i} \in k$, we may choose any $\xi_{1}, \xi_{2}$ and $\xi_{3} \in k$ and solve for the coefficients of $b_{6}$ :

$$
\begin{gathered}
\gamma_{6}=-\xi_{3} \xi_{4}-\psi_{6}, \quad \gamma_{5}=\xi_{3}^{2}-\xi_{2} \xi_{4}-\psi_{5}, \quad \gamma_{4}=-\xi_{1} \xi_{4}-\xi_{2} \xi_{3}-\psi_{4}, \\
\gamma_{3}=\xi_{2}^{3}-\xi_{0} \xi_{4}-\xi_{1} \xi_{3}+\xi_{2}^{3}-\psi_{3}, \quad \gamma_{2}=-\xi_{0} \xi_{1}-\xi_{1} \xi_{2}-\psi_{2}, \\
\gamma_{1}=-\xi_{1}^{2}-\xi_{0} \xi_{2}^{3}-\psi_{1}, \quad \gamma_{0}=-\xi_{0} \xi_{1}+\xi_{1}^{3}-\psi_{0} .
\end{gathered}
$$

Substituting $\mathrm{b}_{2}, \mathrm{~b}_{4}$, and $\mathrm{b}_{6}$ in $\Delta$, we obtain

$$
\Delta=a^{3} t^{12}+a^{2} t^{10}+t^{3} P_{6}+b^{2} t^{2}+b^{3} .
$$

Finally, note that while $\operatorname{PGL}(2)$ is 3-transitive, we only moved two points (the roots of $b_{2}$ ) to specific locations, so we may use PGL(2) to move one root of $\Delta$ to $t=1$ as well.

A similar argument proves the next proposition.

Proposition 3.1.2. Consider a minimal rational elliptic surface over $\mathbb{P}_{\mathrm{t}}^{1}$ with Weierstrauss equation

$$
y^{2}=x^{3}+b_{2} x^{2}-b_{4} x+b_{6}
$$

where $b_{i}$ are polynomials in $t$ and degree $b_{i} \leq i$. If all of the singular fibres are of multiplicative type, and if $\mathrm{b}_{2}$ has a double root, then up to automorphism of the surface and the base, the discriminant $\Delta$ is a degree-twelve polynomial of the form

$$
\Delta=\mathrm{t}^{12}+\mathrm{lt} \mathrm{t}^{9}+\mathrm{m} \mathrm{t}^{8}-\mathrm{n} \mathrm{t}^{7}+\mathrm{P}_{6},
$$

where $\mathrm{l}, \mathrm{m}, \mathrm{n} \in \mathrm{k}$, such that $\mathrm{n}^{3}=\mathrm{lm}^{3} \neq 0$, and $\mathrm{P}_{6}(\mathrm{t})$ is a polynomial of degree less than or equal to 6. Conversely, given any $\Delta$ of the form 3.1.4 there is a rational elliptic surface with Weierstrass equation 3.1.3 and with roots of $\mathrm{b}_{2}$ at $\infty$. If such a surface exists, we may assume one root of $\Delta$ occurs at $\mathrm{t}=0$ and one occurs at $\mathrm{t}=1$. 
To prove the existence of a rational elliptic surface with all singular fibres of multiplicative type, we simply need to exhibit a degree-twelve polynomial in the normal form with the zeros distributed in the desired way. Conversely, if no such polynomials exist, no surface with the prescribed fibre types exists. This yields the following lemma for non-existence of configurations with fibres of high multiplicity.

Lemma D. A rational elliptic surface with purely multiplicative fibres cannot have three (not necessarily distinct) multiplicative fibres each of multiplicity at least three. In particular, no purely multiplicative configuration may include $3^{3}$, $3^{2} 4,34^{2}, 4^{3}, 3^{2} 5,345,36,37,38,46,56,57,6^{2}, 9,10$, 11, or 12 .

Proof. Since we can move one root to $t=1$, the discriminant $\Delta$ of such a surface can be written in the form:

$$
\begin{aligned}
& (t-1)^{3}(t-c)^{3}(t-d)^{3}\left(t^{3}+e t^{2}+f t+g\right)= \\
& t^{12}+e t^{11}+f t^{10}+\left(g-c^{3}-d^{3}-1\right) t^{9}-\left(c^{3} e+d^{3} e+e\right) t^{8} \\
& -\left(c^{3} f+d^{3} f-f\right) t^{7}+\left(c^{3}-d^{3} g-\left(1+c^{3}\right)\left(g-d^{3}\right)\right) t^{6} \\
& +\left(c^{3} e+\left(1+c^{3}\right) d^{3} e\right) t^{5}+\left(\left(1+c^{3}\right) d^{3} f+c^{3} f\right) t^{4} \\
& +\left(\left(i+c^{3}\right) d^{3} g+c^{3}(g-d)\right) t^{3}-c^{3} d^{3} e t^{2}-c^{3} d^{3} f t-c^{3} d^{3} g .
\end{aligned}
$$

If $\mathrm{b}_{2}$ has distinct roots, $\Delta$ must be of the form

$$
t^{12}+l t^{10}+t^{3} P_{6}^{(t)}+m t^{2}+n b^{3},
$$

so the coefficient on the degree-11 term must vanish. Thus $e=0$, and the polynomial is of the form

$$
t^{12}+f t^{10}+t^{3} P_{6}-c^{3} d^{3} f t-c^{3} d^{3} g .
$$

Since the coefficient on the degree-two term must be nonzero to satisfy the form of Equation (3.1.2), we have a contradiction. Notice that no conditions were imposed on the distinctness of the roots of the polynomial.

Suppose now that $b_{2}$ has a double root. Since the coefficients of the degree11 and degree-10 terms must be zero, we have

$$
e=f=0 .
$$

Thus we have

$$
\Delta=t^{12}+\left(g-c^{3}-d^{3}\right) t^{9}+\left(c^{3} d^{3}-d^{3} g-c^{3} g\right) t^{6}+c^{3} d^{3} g t^{3} .
$$

But since the coefficients of the degree- 8 and degree- 7 terms must be nonzero, no such polynomial satisfying Equation (3.1.4) exists. Again, no conditions were imposed on the distinctness of the roots. 


\subsection{Solutions for Purely Multiplicative Singularities}

Below we list the 77 partitions of 12 using the notation of [4] and [2]. Next to each partition, we either list a polynomial of the form given in Equation (3.1.2) whose zeroes are distributed in the way indicated by the partition, or we indicate that the surface does not exist and give the letter of a lemma which proves its non-existence (often there are several that apply). As mentioned above, our results show that whenever a surface exists with purely multiplicative singularities and a double root of $b_{2}$, one also exists with distinct roots of $b_{2}$. We know of no good proof of this fact other than an appeal to our list below.

In the following list $i$ denotes a root of $x^{2}+1=0$ in the field $k$.

1. $1^{12}(t-1)\left(t^{11}+t^{10}-t^{9}+t-1\right)=t^{12}+t^{10}+t^{9}+t^{2}+t+1$

2. $21^{10}(t-1)^{2}\left(t^{10}-t^{9}+t^{8}+t^{2}+t-1\right)=t^{12}+t^{10}+t^{8}+t^{4}-t^{3}+t^{2}-1$

3. $31^{9} \quad(t-1)^{3}\left(t^{9}+t^{7}+t^{6}+t^{3}-t^{2}+1\right)=t^{12}+t^{10}-t^{7}-t^{5}+t^{2}-1$

4. $2^{2} 1^{8} \quad\left(t^{2}+1\right)^{2}\left(t^{8}-t^{6}+t^{4}-t^{2}+1\right)=t^{12}+t^{10}+t^{2}+1$

5. $\quad 41^{8}(t-1)^{4}\left(t^{8}+t^{7}-t^{6}-t^{3}-t^{2}+t+1\right)=t^{12}+t^{10}+t^{7}-t^{6}-t^{5}+$ $t^{4}-t^{3}+t^{2}+1$

6. $\quad 321^{7}(t-1)^{3}(t+1)^{2}\left(t^{7}+t^{6}+t^{5}-t^{2}+t+1\right)=t^{12}+t^{10}-t^{9}+t^{8}+$ $t^{7}-t^{6}+t^{5}+t^{4}-t^{3}+t^{2}-1$

7. $5 \mathbf{1}^{7} \quad(\mathrm{t}-1)^{5}\left(\mathrm{t}^{7}-\mathrm{t}^{6}+\mathrm{t}^{5}+\mathrm{t}^{4}-\mathrm{t}^{2}+\mathrm{t}-1\right)=\mathrm{t}^{12}+\mathrm{t}^{10}-\mathrm{t}^{8}-\mathrm{t}^{7}-\mathrm{t}^{6}-\mathrm{t}^{3}+\mathrm{t}^{2}+1$

8. $2^{3} 1^{6}(t-1)^{2}\left(t^{2}+1\right)^{2}\left(t^{6}-t^{5}-t^{4}+a t^{3}+t-1\right)$ $=t^{12}+t^{10}+(a+1) t^{9}+(a+1) t^{8}-a t^{6}+(a+1) t^{4}+(a+1) t^{3}+t^{2}-1$ where $a \neq 0, \pm 1$

9. $\quad 421^{6} \quad(t-1)^{4}(t+1)^{2}\left(t^{6}-t^{5}-t^{2}+t-1\right)=t^{12}+t^{10}-t^{9}-t^{7}+t^{6}-t^{5}+t^{2}-1$

10. $3^{2} 1^{6} \quad(t-1)^{3}(t+1)^{3}\left(t^{6}+t^{4}-t^{2}+1\right)=t^{12}+t^{10}-t^{8}-t^{4}+t^{2}-1$

11. $6 \mathbf{1}^{6} \quad(\mathrm{t}-1)^{6}\left(\mathrm{t}^{6}+\mathrm{t}^{4}+\mathrm{t}^{2}+1\right)=\mathrm{t}^{12}+\mathrm{t}^{10}+\mathrm{t}^{9}+\mathrm{t}^{8}+\mathrm{t}^{7}-\mathrm{t}^{6}+\mathrm{t}^{5}+\mathrm{t}^{4}+\mathrm{t}^{3}+\mathrm{t}^{2}+1$

12. $32^{2} 1^{5} \quad(t-1)^{3}\left(t^{2}+1\right)^{2}\left(t^{5}-t^{3}+1\right)=t^{12}+t^{10}-t^{9}-t^{8}-t^{6}-t^{4}-t^{3}+t^{2}-1$

13. $521^{5}(t-1)^{5}(t+i+1)^{2}\left(t^{5}+i t^{4}+t^{3}+i t^{2}+(1+i) t+1\right)$ $=t^{12}+(i-1) t^{10}+i t^{9}+t^{8}-(i+1) t^{7}+(1+i) t^{5}-t^{4}+(i-1) t^{3}+(1-i) t^{2}+i$

14. $431^{5} \quad(t-1)^{4}(t+1)^{3}\left(t^{5}+t^{4}-t^{3}-t-1\right)=t^{12}+t^{10}+t^{9}-t^{8}-t^{4}-t^{3}+t^{2}-1$

15. $71^{5} \quad(t-1)^{7}\left(t^{5}+t^{4}-t^{3}+t^{2}+d t+d\right)=t^{12}+t^{10}+(d-1) t^{8}+t^{7}-$ $d t^{6}+(d-1) t^{5}+t^{4}-(d+1) t^{3}+(d-1) t^{2}-d$ where $d^{3}-d^{2}-1=0$

16. $2^{4} 1^{4} \quad(t-1)^{2}(t+1)^{2}\left(t^{2}+1\right)^{2}\left(t^{4}+t^{2}-1\right)=t^{12}+t^{10}+t^{6}+t^{2}-1$

17. $42^{2} 1^{4} \quad(t-1)^{4}\left(t^{2}+1\right)^{2}\left(t^{4}+t^{3}+t^{2}+t+1\right)=t^{12}-t^{10}-t^{9}+t^{8}+t^{4}-$ $t^{3}-t^{2}+1$ 
18. $3^{2} 21^{4} \quad(t-1)^{3}(t+1)^{3}(t+i)^{2}\left(t^{4}+i t^{3}+t^{2}+i t-1\right)=t^{12}+t^{10}-i t^{9}-$ $t^{8}-t^{4}+i t^{3}+t^{2}-1$

19. $621^{4}(t-1)^{6}(t+1)^{2}\left(t^{4}+t^{3}+t^{2}+t+1\right)=t^{12}+t^{10}-t^{9}+t^{8}+t^{7}+$ $t^{5}+t^{4}-t^{3}+t^{2}+1$

20. $531^{4} \quad(t-1)^{5}\left(t^{3}+1\right)\left(t^{4}-t^{3}+t^{2}-\alpha t+\alpha\right)$

$=t^{12}+t^{10}-i t^{9}+t^{8}+(i-1) t^{6}-t^{4}+i t^{3}-t^{2}-i$

21. $4^{2} 1^{4} \quad\left(t^{4}+1\right)^{2}\left(t^{4}+t^{2}-1\right)=t^{12}+t^{10}+t^{8}-t^{6}-t^{4}+t^{2}-1$

22. $81^{4} \quad(t-1)^{8}\left(t^{4}-t^{3}+t^{2}+t-1\right)=t^{12}+t^{10}-t^{9}+t^{8}+t^{7}+t^{6}+t^{5}+t^{4}+t^{2}-1$

23. $32^{3} 1^{3} \quad(t-1)^{3}(t+1)^{2}\left(t^{2}+i t-1\right)^{2}\left(t^{3}+(1+i) t^{2}+(1-i)^{2} t+1-i\right)$ $=t^{12}+t^{10}+t^{9}+t^{8}+(i-1) t^{7}+(1+i) t^{6}+(i-1) t^{5}-i t^{4}+(1+i) t^{3}-i t^{2}+i-1$

24. $52^{2} 1^{3} \quad(t-1)^{5}\left(t^{2}+i t+1\right)^{2}\left(t^{3}+(i-1) t^{2}+i(1-i) t-i\right.$ $=t^{12}-i t^{10}+t^{9}-(1+i) t^{8}-t^{7}-i t^{6}+t^{5}+(i+1) t^{4}+t^{3}+i t^{2}+i$

25. $4321^{3} \quad(t-1)^{4}(t+1)^{3}(t+i)^{2}\left(t^{3}+(1+i) t^{2}+(1-i) t+1\right.$ $=t^{12}+i t^{10}+t^{9}-i t^{8}-i t^{4}-t^{3}+i t^{2}-1$

26. $721^{3} \quad(t-1)^{7}(t+i)^{2}\left(t^{3}+(i+1) t^{2}+c t-c(i+1)\right)$

$=t^{12}+(c-i-1) t^{10}+(i c+c) t^{9}+(1-c+i) t^{8}+(c-i-1) t^{7}+(1-c+$ i) $t^{5}+(c-i-1) t^{4}-t^{3}+(1-c+i) t^{2}-c-i c$

where $i c^{5}-c^{3}-(i+1) c^{2}-i+1=0$

27. $\quad \mathbf{3}^{\mathbf{3}} \mathbf{1}^{\mathbf{3}}$ does not exist, D

28. $63 \mathbf{1}^{3}$ does not exist, D

29. $541^{3} \quad\left((t-1)^{5}(t+i)^{4}\left(t^{3}-(1+i) t^{2}+t-i-1\right)\right.$

$=t^{12}-(i-1) t^{10}+t^{9}-(i+1) t^{8}-i t^{7}+(1-i) t^{6}-i t^{5}+(1-i) t^{4}+(1-$ i) $t^{3}+(1-i) t^{2}-1-i$

30. $\quad 91^{3}$ does not exist, $\mathbb{D}$

31. $\mathbf{2}^{5} \mathbf{1}^{2} \quad\left(\mathrm{t}^{5}-1\right)^{2}\left(\mathrm{t}^{2}+1\right)=\mathrm{t}^{12}+\mathrm{t}^{10}+\mathrm{t}^{7}+\mathrm{t}^{5}+\mathrm{t}^{2}+1$

32. $\quad 42^{3} 1^{2} \quad(t-1)^{4}\left(t^{2}+t+1\right)^{2}(t+1)^{2}\left(t^{2}+1\right)=t^{12}-t^{10}+t^{9}-t^{8}-t^{7}-$ $t^{6}-t^{5}-t^{4}+t^{3}-t^{2}+1$

33. $3^{2} 2^{2} 1^{2}(t-1)^{3}(t+1)^{3}\left(t^{2}+i\right)^{2}\left(t^{2}+i+1\right)$ $=t^{12}+t^{10}-i t^{8}+(1-i) t^{6}-t^{4}+i t^{2}+i+1$

34. $62^{2} 1^{2} \quad(t-1)^{6}\left(t^{2}+1\right)^{2}\left(t^{2}+i\right)$ $=t^{12}+(i-1) t^{10}+t^{9}+(1-i) t^{8}+(i-1) t^{7}+(i+1) t^{6}+(1-i) t^{5}+(i-$ 1) $t^{4}+i t^{3}+(1-i) t^{2}+i$

35. $5321^{2} \quad(t-1)^{5}\left(t^{3}+i\right)(t+1)^{2}\left(t^{2}-1-i\right)$ $=t^{12}-i t^{10}+(i-1) t^{9}-i t^{8}+(1+i) t^{7}+(i-1) t^{6}+(1+i) t^{5}-t^{4}-t^{3}-t^{2}+i-1$

36. $4^{2} 21^{2} \quad(t-1)^{4}(t-i)^{4}(t+1-i)^{2}\left(t^{2}-t+i\right)$ $=t^{12}+t^{10}+i t^{9}+i t^{8}+(i-1) t^{7}-(1+i) t^{6}-(1+i) t^{5}-i t^{4}+t^{3}+t^{2}-1$ 
37. $821^{2} \quad(t-1)^{8}(t+1)^{2}\left(t^{2}+1\right)=t^{12}-t^{10}+t^{9}-t^{8}-t^{7}-t^{6}-t^{5}-t^{4}+t^{3}-t^{2}+1$

38. $\quad \mathbf{4 3}^{\mathbf{2}} \mathbf{1}^{\mathbf{2}}$ does not exist, [C]

39. $\quad \mathbf{7 3} \mathbf{1}^{\mathbf{2}}$ does not exist, $\mathrm{B}$

40. $\mathbf{6 4 1 ^ { 2 }}$ does not exist, $\mathrm{C}$

41. $5^{2} \mathbf{1}^{2}(t-1)^{5}(t+1)^{5}\left(t^{2}+i\right)$

$=t^{12}+(1+i) t^{10}+(1+i) t^{8}+(i-1) t^{6}-(1+i) t^{4}-(1+i) t^{2}-i$

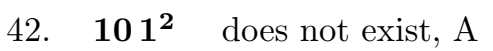

43. $32^{4} 1 \quad(t-1)^{3}\left(t^{4}+t^{3}-t^{2}-t-1\right)^{2}(t+1)=t^{12}+t^{10}-t^{8}-t^{7}-t^{6}+t^{3}+t^{2}-1$

44. $\quad 5 \mathbf{2}^{3} \mathbf{1}$ does not exist, $\mathrm{C}$

45. $432^{2} 1 \quad(t-1)^{4}\left(t^{3}-i\right)\left(t^{2}+c\right)^{2}(t+1)$

$=t^{12}-(1+c) t^{10}+(i-1) t^{9}+\left(c^{2}+c\right) t^{8}+(c-i+1-i c) t^{7}-\left(c^{2}+i\right) t^{6}+$ $\left(i c^{2}-c^{2}+i c-c\right) t^{5}+(i c+i) t^{4}+\left(c^{2}-i c^{2}\right) t^{3}-\left(i c+i c^{2}\right) t^{2}+i c^{2}$

where $c^{4}-i c^{3}+c-i=0$

46. $\mathbf{7 2}^{\mathbf{2}} \mathbf{1}$ does not exist, B

47. $\mathbf{3}^{\mathbf{3}} \mathbf{2} 1$ does not exist, $\mathrm{D}$

48. 6321 does not exist, D

49. 5421 does not exist, B

50. 921 does not exist, A

51. $\quad \mathbf{5} \mathbf{3}^{\mathbf{2}} \mathbf{1}$ does not exist, $\mathrm{C}$

52. $\mathbf{4}^{2} \mathbf{3} 1$ does not exist, $\mathrm{C}$

53. $\mathbf{8 3 1}$ does not exist, $\mathrm{A}$

54. $\mathbf{7 4 1}$ does not exist, A

55. 651 does not exist, A

56. 111 does not exist, $\mathrm{A}$

57. $2^{6} \quad(t-1)^{2}\left(t^{5}+t^{4}+t+1\right)^{2}=t^{12}+t^{10}-t^{6}+t^{2}+1$

58. $\quad 42^{4} \quad(t-1)^{4}\left(t^{4}-t^{3}+t^{2}-t+1\right)^{2}=t^{12}-t^{10}+t^{9}+t^{7}-t^{6}+t^{5}+t^{3}-t^{2}+1$

59. $\quad \mathbf{3}^{\mathbf{2}} \mathbf{2}^{\mathbf{3}}$ does not exist, $\mathrm{C}$

60. $\quad \mathbf{6 2}^{\mathbf{3}}$ does not exist, $\mathrm{C}$

61. $\quad \mathbf{5} 3 \mathbf{2}^{\mathbf{2}}$ does not exist, $\mathrm{C}$

62. $4^{2} 2^{2} \quad(t-1)^{4}(t+1)^{4}\left(t^{2}+1\right)^{2}=t^{12}+t^{10}-t^{8}+t^{6}-t^{4}+t^{2}+1$

63. $\quad \mathbf{8 2}^{\mathbf{2}}$ does not exist, $\mathrm{A}$

64. $\quad \mathbf{4 3}^{2} \mathbf{2}$ does not exist, B 

65. $\mathbf{7 3 2}$ does not exist, $\mathrm{A}$
66. 642 does not exist, A
67. $\quad \mathbf{5}^{2} \mathbf{2}$ does not exist, $\mathrm{A}$
68. 102 does not exist, $\mathrm{A}$
69. $\mathbf{3}^{\mathbf{4}}$ does not exist, D
70. $\mathbf{6 ~}^{\mathbf{2}}$ does not exist, $\mathrm{A}$
71. $\mathbf{5 4 3}$ does not exist, A
72. 93 does not exist, $\mathrm{A}$
73. $\quad \mathbf{4}^{\mathbf{3}}$ does not exist, $\mathrm{A}$
74. 84 does not exist, \$
75. 75 does not exist, $\mathrm{A}$
76. $\mathbf{6}^{\mathbf{2}}$ does not exist, A
77. 12 does not exist, A

\section{At Least One Additive Fibre}

We now consider the case where the rational elliptic surface has at least one additive fibre.

\subsection{General Information}

In each of the cases below we will use a linear transformation to send the worst singularity to $t=0$, using the fact that PGL(2) acts three-transitively on $\mathbb{P}^{1}$. We classify "worst" singularities using the following criteria:

1. Additive type singularities are worse than multiplicative type singularities.

2. If comparing two additive type singularities with different values of $\delta$ (where $\delta$ is the multiplicity of the root of $\Delta$ over which the singularity happens), the singularity with the largest $\delta$ is worse.

3. If two additive type singularities have the same value of $\delta$, the singularity with the largest $r$ value is worse.

As in [3, we may change coordinates so that the Weierstrass equation becomes

$$
y^{2}=x^{3}+t c_{1} x^{2}+t_{3} x+t c_{5}
$$

where the $c_{i}$ are polynomials in $t$ of degree no more than $i$. In our notation,

$$
\mathrm{b}_{2}=\mathrm{tc}_{1}, \quad \mathrm{~b}_{4}=-\mathrm{tc}_{3}, \quad \text { and } \mathrm{b}_{6}=\mathrm{tc}_{5},
$$


and $\Delta$ becomes

$$
-\Delta=t^{4} c_{1}^{2}\left(c_{1} c_{5}-c_{3}^{2}\right)+t^{3} c_{3}^{3} .
$$

A list of all singular fibres of additive type that could potentially appear on a rational elliptic surface in characteristic three was given in [3], and a normal form over $t=0$ was given for each one. We will use the normal forms of [3] for each of the worst singularities. For the reader's convenience, these normal forms are listed at the beginning of each case treated in Section 4.2

An easy application of Tate's algorithm (or a glance at Equation (4.1.2) shows that all additive fibres have $\delta \geq 3$. The following lemma is also an easy result of Tate's algorithm [9].

Lemma $\mathbf{E}$. If $\mathrm{b}_{2} \equiv 0$, then all singular fibres on the surface are of additive type. If $\mathrm{t}^{2} \mid \mathrm{b}_{2}$, where $\mathrm{b}_{2}$ is not identically zero, then there is only one additive singular fibre.

Since PGL(2) is three-transitive, after sending the worst singularity to $t=0$, we may still move two other points of the base either singular fibres or roots of $b_{2}$.

As in the purely multiplicative situation, we may consider two cases: when $b_{2}$ has a double root at $t=0$, and when $b_{2}$ has distinct roots. If $b_{2}$ has distinct roots, we can move the second root of $b_{2}$ to $t=\infty$. Again, this gives some significant restrictions on $\Delta$.

Proposition 4.1.1. Consider a minimal rational elliptic surface over $\mathbb{P}_{t}^{1}$ with a singular fibre of additive type over $\mathrm{t}=0$ and Weierstrass equation

$$
\mathrm{y}^{2}=\mathrm{x}^{3}+\mathrm{tc}_{1} \mathrm{x}^{2}+\mathrm{tc}_{3} \mathrm{x}+\mathrm{tc}_{5}
$$

where the $\mathrm{c}_{\mathrm{i}}$ are polynomials in $\mathrm{t}$ of degree no more than $\mathrm{i}$. If the roots of $\mathrm{b}_{2}$ are distinct, then up to automorphism of the surface and the base, the discriminant of the surface is a polynomial of degree 12 or less, of the form

$$
\Delta=-\alpha^{3} t^{12}+\alpha^{2} \beta^{2} t^{10}+t^{6} P_{3}(t)+\beta^{2} t^{4} P_{1}(t)-\sigma^{3} t^{3},
$$

where $\alpha, \beta$, and $\sigma$ are elements of $k$, and the $P_{i}(t)$ are elements of $k[t]$ of degree no more than $\mathrm{i}$. In particular, the $\mathrm{t}^{11}$ term vanishes.

Similarly, if $\mathrm{b}_{2}$ has a double root, then up to automorphism of the surface and the base, the discriminant of the surface is a polynomial of degree 12 or less, of the form

$$
\Delta=\mathrm{t}^{6} \mathrm{P}_{6}(\mathrm{t})-\sigma \mathrm{t}^{3}
$$

where $\sigma$ is an element of $\mathrm{k}$, and $\mathrm{P}_{6}$ is an element of $\mathrm{k}[\mathrm{t}]$ of degree no more than 6. In particular, the $\mathrm{t}^{4}$ and $\mathrm{t}^{5}$ terms vanish.

Finally, if $\mathrm{b}_{2}$ is identically zero, then up to automorphism of the surface and the base, the discriminant of the surface is a polynomial of degree 12 or less, of the form

$$
\Delta=\mathrm{b}_{4}^{3}=-\mathrm{t}^{3}\left(\mathrm{c}_{3}\right)^{3} .
$$


Proof. We let

$$
\begin{array}{cl}
\mathrm{b}_{2}=\mathrm{tc}_{1} & =\mathrm{t}\left(\epsilon_{1} \mathrm{t}+\epsilon_{0}\right) \\
\mathrm{b}_{4}=-\mathrm{tc}_{3} & =\mathrm{t}\left(\phi_{3} \mathrm{t}^{3}+\phi_{2} \mathrm{t}^{2}+\phi_{3} \mathrm{t}+\phi_{0}\right) \\
\mathrm{b}_{6}=\mathrm{tc}_{5} & =\mathrm{t}\left(\gamma_{5} \mathrm{t}^{5}+\gamma_{4} \mathrm{t}^{4}+\cdots+\gamma_{0}\right),
\end{array}
$$

with $\epsilon_{i}, \phi_{i}$ and $\gamma_{i}$ in $k$. If $b_{2}$ has distinct roots, then we may move the second root to infinity, which amounts to setting $\epsilon_{1}=0$. We substitute these into the expression (4.1.2) for $\Delta$ to get

$$
\begin{aligned}
\Delta & =-\phi_{3}^{3} t^{12}+\epsilon_{0}^{2} \phi_{3}^{2} t^{10}-\left(\epsilon_{0}^{2} \phi_{3} \phi_{2}+\epsilon_{0}^{3} \gamma_{5}+\phi_{2}^{3}\right) t^{9} \\
& +\left(2 \epsilon_{0}^{3} \gamma_{4}+\epsilon_{0}^{2} \phi_{2}^{2}+2 \epsilon_{0}^{2} \phi_{3} \phi_{1}\right) t^{8} \\
& -\left(\epsilon_{0}^{3} \gamma_{3}+\epsilon_{0}^{2} \phi_{2} \phi_{1}+\epsilon_{0}^{2} \phi_{3} \phi_{0}\right) t^{7} \\
& +\left(\epsilon_{0}^{2} \phi_{1}^{2}+2 \phi_{1}^{3}+2 \epsilon_{0}^{2} \phi_{2} \phi_{0}+2 \epsilon_{0}^{3} \gamma_{2}\right) t^{6} \\
& +\left(2 \epsilon_{0}^{3} \gamma_{1}+2 \epsilon_{0}^{2} \phi_{1} \phi_{0}\right) t^{5}+\left(2 \epsilon_{0}^{3} \gamma_{0}+\epsilon_{0}^{2} \phi_{0}^{2}\right) t^{4}+2 t^{3} \phi_{0}^{3} .
\end{aligned}
$$

This has the desired form.

Similarly, if $b_{2}$ has a double root, we may assume that $\epsilon_{0}=0$, and substituting into Equation (4.1.2) gives

$$
\begin{array}{r}
\Delta=\quad\left(\epsilon_{1}^{2} \phi_{3}^{2}+2 \epsilon_{1}^{3} \gamma_{5}+2 \phi_{3}^{3}\right) t^{12}+\left(2 \epsilon_{1}^{3} \gamma_{4}+2 \epsilon_{1}^{2} \phi_{3} \phi_{2}\right) t^{11} \\
+\left(2 \epsilon_{1}^{2} \phi_{3} \phi_{1}+\epsilon_{1}^{2} \phi_{2}^{2}+2 \epsilon_{1}^{3} \gamma_{3}\right) t^{10} \\
+\left(2 \epsilon_{1}^{2} \phi_{2} \phi_{1}+2 \epsilon_{1}^{2} \phi_{3} \phi_{0}+2 \epsilon_{1}^{3} \gamma_{2}+2 \phi_{2}^{3}\right) t^{9} \\
+\left(2 \epsilon_{1}^{2} \phi_{2} \phi_{0}+2 \epsilon_{1}^{3} \gamma_{1}+\epsilon_{1}^{2} \phi_{1}^{2}\right) t^{8} \\
+\left(2 \epsilon_{1}^{3} \gamma_{0}+2 \epsilon_{1}^{2} \phi_{1} \phi_{0}\right) t^{7}+\left(\epsilon_{1}^{2} \phi_{0}^{2}+2 \phi_{1}^{3}\right) t^{6}+2 t^{3} \phi_{0}^{3} .
\end{array}
$$

This again has the desired form.

Finally, substituting $b_{2}=0$ into Equation (4.1.2) gives the last form.

An immediate corollary of the proposition is the following lemma.

Lemma $\mathbf{F}$. The configuration $\mathbf{X} \mathbf{3}^{\mathbf{2}} \mathbf{2} \mathbf{1}$ is not possible, where $\mathbf{X}$ is any additive fibre. Nor is any specialization of this configuration possible, provided the $\mathbf{I}_{\mathbf{2}}$ and $\mathbf{I}_{\mathbf{1}}$ fibres remain distinct from each other and both the order-three multiplicative singularities remain distinct from the additive fibre $\mathbf{X}$. More precisely, the following configurations cannot exist: X $3^{2} 21, \mathrm{X} 621, \mathrm{X} 531, \mathrm{X} 432$, X 54, $\mathrm{X}^{2} 1, \mathrm{X} 61, \mathrm{X}^{2} 2, \mathrm{X} 62, \mathrm{X} 43, \mathrm{X} 53$.

Proof. Assume this configuration exists. $X$ must have $\delta=3$, since $\sum \delta_{i}=12$, and we may assume $X$ occurs over $t=0$. We may further assume no singular fibre occurs over infinity. We let the first $I_{3}$ occur over $t=a$, the second $I_{3}$ over $\mathrm{t}=\mathrm{b}$, the $\mathrm{I}_{2}$ over $\mathrm{t}=\mathrm{c}$, and the $\mathrm{I}_{1}$ over $\mathrm{t}=\mathrm{d}$.

The hypotheses of the lemma require $c \neq d$, and $a b \neq 0$ but do not otherwise require that the roots be distinct or non-vanishing. We have $\eta t^{3}(t-a)^{3}(t-$ 
$b)^{3}(t-c)^{2}(t-d)=\Delta$ for some $\eta \in k^{*}$. We expand to get

$$
\begin{gathered}
t^{3}(t-a)^{3}(t-b)^{3}(t-c)^{2}(t-d)= \\
t^{12}+(c+2 d) t^{11}+\left(2 c d+c^{2}\right) t^{10} \\
+\left(2 b^{3}+2 c^{2} d+2 a^{3}\right) t^{9}+\left(2 a^{3} c+2 b^{3} c+a^{3} d+b^{3} d\right) t^{8} \\
+\left(2 b^{3} c^{2}+a^{3} c d+b^{3} c d+2 a^{3} c^{2}\right) t^{7} \\
+\left(a^{3} b^{3}+a^{3} c^{2} d+b^{3} c^{2} d\right) t^{6}+\left(2 a^{3} b^{3} d+a^{3} b^{3} c\right) t^{5} \\
+\left(2 a^{3} b^{3} c d+a^{3} b^{3} c^{2}\right) t^{4}+2 t^{3} a^{3} b^{3} c^{2} d
\end{gathered}
$$

If $b_{2}$ has distinct roots, the $t^{11}$ term of $\Delta$ must vanish, as in equation (4.1.4), which means $\mathrm{c}=\mathrm{d}$, a contradiction.

If $b_{2}$ has a repeated root, then the $t^{5}$ term of $\Delta$ must vanish, as in equation (4.1.5), which means $a^{3} b^{3} c=a^{3} b^{3} d$, and thus either $c=d$ or $a b=0$, a contradiction.

All of the configurations that do not exist fail to do so by one of the previous lemmas, or by straightforward algebraic manipulations. We list all that fail from algebraic manipulations in the following lemma.

Lemma G. The following configurations do not exist:

1. II $3^{2}$ and II 6 .

2. IV $\mathbf{2}^{3}$ and IV 42.

3. IV 321 and IV 51.

4. $\quad \mathbf{I}_{1}^{*} 41$.

5. III $4^{2} 1$ and III 54.

6. III III $3^{2}$, III III 6, III II $3^{2}$, and III II 6 .

7. III III 42 , and III II 42 , where the $\mathrm{I}_{4}$ and $\mathrm{I}_{2}$ are distinct.

Proof. 1. II $\mathbf{3}^{\mathbf{2}}$ and II 6. Suppose this configuration exists. Assume that one $I_{3}$ singularity occurs over $t=a$ and the other over $t=b$. We allow the possibility of $a=b$. The fibre of type II must have $\delta=6$, which means it is case 1C, which requires that $\epsilon_{0}=0, \phi_{0}=0$, and $\gamma_{0} \neq 0$ (see Section 4.2.3). Making the substitutions of Equation (4.1.7) for $b_{i}$ in the discriminant, we have

$$
\begin{aligned}
\alpha\left[(\mathrm{t}-\mathrm{a})^{3}(\mathrm{t}-\mathrm{b})^{3}\right]= & -\Delta / \mathrm{t}^{6} \\
= & \left(2 \phi_{3}^{3}+2 \epsilon_{1}^{3} \gamma_{5}+\epsilon_{1}^{2} \phi_{3}^{2}\right) \mathrm{t}^{6}+\left(2 \epsilon_{1}^{2} \phi_{3} \phi_{2}+2 \epsilon_{1}^{3} \gamma_{4}\right) \mathrm{t}^{5} \\
& +\left(\epsilon_{1}^{2} \phi_{2}^{2}+2 \epsilon_{1}^{3} \gamma_{3}+2 \epsilon_{1}^{2} \phi_{3} \phi_{1}\right) \mathrm{t}^{4} \\
& +\left(2 \epsilon_{1}^{3} \gamma_{2}+2 \epsilon_{1}^{2} \phi_{2} \phi_{1}+2 \phi_{2}^{3}\right) \mathrm{t}^{3} \\
& +\left(\epsilon_{1}^{2} \phi_{1}^{2}+2 \epsilon_{1}^{3} \gamma_{1}\right) \mathrm{t}^{2}+2 \mathrm{t} \epsilon_{1}^{3} \gamma_{0}+2 \phi_{1}^{3},
\end{aligned}
$$


for some $\alpha \in k$.

Since the right-hand side of the above equation has a $t$ term, but the left-hand side does not, the $t$ term must vanish. But $\epsilon_{1}=0$ would force $\mathrm{b}_{2}=0$, and thus all fibres would be additive, and $\gamma_{0}=0$ is prohibited in case $1 \mathrm{C}$.

2. IV $\mathbf{2}^{\mathbf{3}}$ and IV 4 2. Suppose this configuration exists. Since the type IV fibre occurs with $\delta=6$, we are in case $3 \mathrm{C}$, with $\epsilon_{1}=0, \phi_{0}=0, \gamma_{0}=0$, but $\phi_{1} \neq 0$. Assume the multiplicative fibres occur over $a, b$, and $c$ in $\mathbb{A}^{1}-\{0\}$. If all $\mathrm{a}=\mathrm{b}=\mathrm{c}$, we have the configuration IV 6, which is possible (see Section 4.2.9). If any two of these fibres are distinct, we may assume, without loss of generality, that $a=1$ and $b=-1$, which means we have

$$
\begin{gathered}
\alpha\left[t^{6}+c t^{5}+\left(1+c^{2}\right) t^{4}+c t^{3}+\left(1+c^{2}\right) t^{2}+c t+c^{2}\right]=-\Delta / t^{6} \\
=\left(-\epsilon_{1}^{3} \gamma_{5}+\epsilon_{1}^{2} \phi_{3}^{2}-\phi_{3}^{3}\right) t^{6}+\left(2 \epsilon_{1}^{3} \gamma_{4}-\epsilon_{1}^{2} \phi_{3} \phi_{2}\right) t^{5}+\left(\epsilon_{1}^{2} \phi_{2}^{2}-\epsilon_{1}^{2} \phi_{3} \phi_{1}-\epsilon_{1}^{3} \gamma_{3}\right) t^{4} \\
+\left(2 \phi_{2}^{3}-\epsilon_{1}^{2} \phi_{2} \phi_{1}-\epsilon_{1}^{3} \gamma_{2}\right) t^{3}+\left(2 \epsilon_{1}^{3} \gamma_{1}+\epsilon_{1}^{2} \phi_{1}^{2}\right) t^{2}-\phi_{1}^{3} .
\end{gathered}
$$

Comparing low-degree coefficients shows that $c=0$, a contradiction.

3. IV 32 1, IV 5 1. Suppose this configuration exists. Again the IV falls in case $3 \mathrm{C}$, and we assume the $\mathrm{I}_{3}$ fibre occurs over $\mathrm{a}$, the $\mathrm{I}_{2}$ over $\mathrm{b}$, and the $I_{1}$ over $c$, with $a b c \neq 0$. If the $I_{2}$ and $I_{1}$ fibres are distinct, we have $b \neq c$, and we may assume that $b=1$ and $c=-1$, which yields the following:

$$
\begin{gathered}
\alpha\left[t^{6}+2 t^{5}+2 t^{4}+\left(1+2 a^{3}\right) t^{3}+a^{3} t^{2}+a^{3} t+2 a^{3]}=-\Delta / t^{6}\right. \\
=\left(-\epsilon_{1}^{3} \gamma_{5}+\epsilon_{1}^{2} \phi_{3}^{2}-\phi_{3}^{3}\right) t^{6}+\left(2 \epsilon_{1}^{3} \gamma_{4}-\epsilon_{1}^{2} \phi_{3} \phi_{2}\right) t^{5}+\left(\epsilon_{1}^{2} \phi_{2}^{2}-\epsilon_{1}^{2} \phi_{3} \phi_{1}-\epsilon_{1}^{3} \gamma_{3}\right) t^{4} \\
+\left(2 \phi_{2}^{3}-\epsilon_{1}^{2} \phi_{2} \phi_{1}-\epsilon_{1}^{3} \gamma_{2}\right) t^{3}+\left(2 \epsilon_{1}^{3} \gamma_{1}+\epsilon_{1}^{2} \phi_{1}^{2}\right) t^{2}-\phi_{1}^{3} .
\end{gathered}
$$

Comparing low-degree coefficients shows that $a=0$, a contradiction.

4. $\mathbf{I}_{\mathbf{1}}^{*} \mathbf{4}$ 1. Suppose this configuration exists. The $\mathbf{I}_{\mathbf{1}}^{*}$ fibre falls into case $5 A$, and thus has $\phi_{0}=\phi_{1}=\phi_{2}=0$ and $\gamma_{0}=\gamma_{1}=\gamma_{2}=0$, but $\epsilon_{0} \neq 0$ and $\gamma_{3} \neq 0$. In particular, $b_{2}$ has distinct roots. We may assume that the non-zero root of $b_{2}$ occurs at infinity, which implies that $\epsilon_{1}=0$, and the $I_{4}$ fibre occurs at $t=1$. The remaining $I_{1}$ fibre occurs over some $a \in \mathbb{A}^{1}-\{0,1, \infty\}$. Making these substitutions, we have

$$
\begin{aligned}
\alpha\left[t^{5}-(1+a) t^{4}+t^{3} a-t^{2}\right. & +(1+a) t-a]=\Delta / t^{7} \\
& =2 \phi_{3}{ }^{3} t^{5}+\phi_{3}{ }^{2} t^{3}-t^{2} \gamma_{5}+2 t \gamma_{4}+2 \gamma_{3} .
\end{aligned}
$$

Since the coefficient of $t^{4}$ vanishes in $\Delta / t^{7}$, we have that $a=1$, which is a contradiction.

5. III $4^{2} 1$ and III 5 4. Suppose this configuration exists. The type III fibre falls into case 2 , which means that $\gamma_{0}=0$, but $\phi_{0} \neq 0$ and $\epsilon_{0} \neq 0$. Let 
the $I_{4}$ 's happen at $t=a$ and $t=b$, and let the $I_{1}$ happen at $t=c . W e$ require that $a \neq b$, but we could have $a=c$ or $b=c$. We have

$$
\Delta=\alpha\left(t^{3}(t-a)^{4}(t-b)^{4}(t-c)\right)
$$

for some $\alpha \neq 0$. If $b_{2}$ has two distinct roots, then by Proposition 4.1.1 we may assume that $\epsilon_{1}=0$ and $\Delta$ has no $t^{11}$ term. This means $c=-a-b$. Furthermore, we may assume that $a=1$. Making these substitutions into $\Delta$ shows that the leading term vanishes if $\phi_{3}$ does, which would put an additional additive singularity at infinity. Thus $\phi_{3}$ is non-zero. Equating like terms in Equation (4.1.10), we obtain the following:

$$
\begin{array}{rlrl}
\frac{\phi_{0}^{3}}{\phi_{3}^{3}} & =b^{5}+b^{4} & \left(\text { from } t^{3}\right) \\
-\frac{\epsilon_{0}^{2} \phi_{0}^{2}}{\phi_{3}^{3}} & =-b^{4}-b^{3}-b^{5} & & \left(\text { from } t^{4}\right) \\
-\frac{\epsilon_{0}^{2}}{\phi_{3}} & =-1-b-b^{2} & & \left(\text { from } t^{10}\right) .
\end{array}
$$

A straightforward manipulation of these constraints gives $b=1$, which is a contradiction, since $\mathrm{a}=1$ and $\mathrm{a}$ and $\mathrm{b}$ are distinct.

If $b_{2}$ has a repeated root, by Proposition 4.1.1 the $t^{5}$ and $t^{4}$ terms must vanish, and we may assume that $a=1$ and $b=-1$. Substituting into Equation (4.1.10), and setting like terms equal, we get that $c=0$, which is a contradiction.

6. III III $3^{2}$, III III 6, III II $3^{2}$, and III II 6 .

Suppose this configuration is possible. We may assume that the type III fibre occurs over $t=0$, which implies that $\phi_{0} \neq 0$, but $\gamma_{0}=0$. We further assume that the other additive fibre occurs over $t=\infty$, so that $b_{2}$ has distinct roots, and we may take $\epsilon_{0}=1$ and $\epsilon_{1}=0$. Furthermore, suppose the first $I_{3}$ happens at $t=a$ for some $a \neq 0, \infty$, and that the second $I_{3}$ happens at $\mathrm{t}=\mathrm{b} \neq 0, \infty$. Making these substitutions into $\Delta$ shows that the coefficient of $t^{4}$ in $\Delta$ is $\phi_{0}^{2}$. But matching coefficients in the equality

$$
\Delta=\alpha t^{3}(t-a)^{3}(t-b)^{3}=t^{9}-\left(a^{3}+b^{3}\right) t^{6}-t^{3} a^{3} b^{3},
$$

with $\alpha \in k^{*}$, shows that $\phi_{0}=0$, which is a contradiction.

7. III III 42 and III II 42. Suppose this configuration is possible. As in the previous case, we may assume that $\gamma_{0}=\epsilon_{1}=0$ and $\epsilon_{0}=1$. Since the $t^{8}$ term vanishes in the expansion of $\Delta$, matching coefficients in the equality

$$
\Delta=\alpha(\mathrm{t}-\mathrm{a})^{4}(\mathrm{t}-\mathrm{b})^{2}
$$

shows that $a=b$, which is a contradiction. 


\subsection{Solutions for Additive and Multiplicative Singularities}

We list Lang's type [3] for the worst singularity, its main invariants, Weierstrass form, and $-\Delta$. For each subsequent configuration, we list the Weierstrass form and $-\Delta$ if the desired rational elliptic surface exists, or the lemma number proving impossibility if it does not exist. In the following examples, $i$ denotes a root of $x^{2}+1=0$ in $k$.

\subsubsection{Case 1A}

$$
\begin{gathered}
\delta=3, \quad r=0 \quad \text { Type II } \\
y^{2}=x^{3}+t c_{1} x^{2}+t c_{3} x+t c_{5}, \quad t \nmid c_{5}, t \nmid c_{3} \\
-\Delta=t^{2} c_{1}^{2}\left(t^{2} c_{1} c_{5}-t^{2} c_{3}^{2}\right)+t^{3} c_{3}^{3}
\end{gathered}
$$

1. II 9 $y^{2}=x^{3}+t^{2} x^{2}+t(t+1) x+t(t+2)$

$-\Delta=2 \mathrm{t}^{3}$

2. II 81 does not exist, $\mathbb{E}$

3. II 72 does not exist, $\mathbb{E}$

4. II $7 \mathbf{1}^{2} \quad y^{2}=x^{3}+t(t+i) x^{2}+t(2 t+i) x+t^{2}$

$-\Delta=-2 i t^{3}+t^{4}-2 i t^{5}=-t^{3}\left(2+i t+2 t^{2}\right) i$

5. II $63 \quad y^{2}=x^{3}+t^{2} x^{2}+t(2 t+2) x+t(2+t)$

$-\Delta=t^{9}+2 t^{6}=t^{6}\left(t^{3}+2\right)$

6. II 621 does not exist, $\mathbb{E}$

7. II $61^{3} \quad y^{2}=x^{3}+t(t+1) x^{2}+t(t+2+i) x+t(t+2 i)$

$-\Delta=(-1-i) t^{6}+i t^{5}-i t^{4}+(1+i) t^{3}=-t^{3}(t-1)\left((1+i) t^{2}+t+(1+i)\right)$

8. II 5 does not exist, $\mathbf{F}$

9. II 531 does not exist, $\mathbf{E}$

10. II 5 2 $2^{2} \quad y^{2}=x^{3}+t \epsilon_{0} x^{2}+t\left(2 t^{3}+2+i\right) x+\frac{t}{\epsilon_{0}}\left((2+2 i) t^{5}+2 i t^{4}+(2+\right.$ i) $\left.\mathrm{t}^{3}+\mathrm{t}^{2}+(2+2 i) \mathrm{t}+2 \mathrm{i}\right)$

$-\Delta=t^{12}+i t^{10}+(-1+i) t^{9}+2 t^{8}+2 i t^{6}+(-1+i) t^{5}+t^{4}+(1+i) t^{3}=$ $t^{3}(t+(1+i))^{5}(t-1)^{2}(t-i)^{2}$, where $\epsilon_{0}^{2}=i$

11. II $521^{2} \quad y^{2}=x^{3}+t x^{2}+t\left(2 t^{3}+i\right) x+t\left(i t^{5}+t^{4}+2 i t^{3}+t^{2}+2 i t+2\right)$ $-\Delta=t^{12}+t^{10}+2 i t^{9}+2 t^{8}+2 i t^{7}+2 t^{6}+i t^{5}+i t^{3}=t^{3}(t+i)^{5}(t-i)^{2}(t-$ 1) $(t+1)$

12. II $51^{4} \quad y^{2}=x^{3}+t \epsilon_{0} x^{2}+t\left(2 t^{3}+i\right) x+\frac{t}{\epsilon_{0}}\left((1-i) t^{5}+(1+i) t^{4}+t^{3}+\right.$ $\left.(1+i) t^{2}+i t-i\right)$

$-\Delta=t^{12}+(1+i) t^{10}+t^{9}+i t^{8}+t^{7}+i t^{6}+(1+2 i) t^{5}+t^{4}+i t^{3}=$ $t^{3}(t-(1-i))^{5}(t-i)(t+1)(t-1)(t-(1+i))$ 
13. II $4^{2} 1 \quad y^{2}=x^{3}+t(t+\epsilon) x^{2}+t x+t\left(\frac{-t}{\epsilon-1}+\frac{\epsilon}{\epsilon-1}\right)$

$-\Delta=\frac{\mathrm{t}^{8}}{\epsilon-1}-\frac{\epsilon \mathrm{t}^{7}}{\epsilon-1}+\mathrm{t}^{6}-\frac{\mathrm{t}^{5}}{\epsilon-1}+\frac{\epsilon \mathrm{t}^{4}}{\epsilon-1}+\frac{(1-\epsilon) \mathrm{t}^{3}}{\epsilon-1}=\mathrm{t}^{3}(\mathrm{t}-1)^{4}\left(2+\frac{1}{\epsilon-1}-\frac{\epsilon}{\epsilon-1}-1+\epsilon^{2}\right) \mathrm{t}$, where $\epsilon^{3}=\epsilon^{2}-\epsilon-1$

14. II 432 does not exist, F

15. II $431^{2} \quad y^{2}=x^{3}+t(t+1) x^{2}+t x+t(t+2)$

$-\Delta=2 t^{8}+t^{7}+t^{6}+t^{5}+2 t^{4}+2 t^{3}=-t^{3}(t-1)^{3}\left(2-t+t^{2}\right)$

16. II $42^{2} 1 \quad y^{2}=x^{3}+t x^{2} i+t\left(2 t^{3}+2 i\right) x+t\left(2 t^{5}+i t^{4}-i t^{2}+t+i\right)$

$-\Delta=t^{12}+2 t^{10}-i t^{9}+2 t^{8}+i t^{7}+t^{6}+i t^{5}+2 i t^{3}=t^{3}(t+i)^{4}(t-1)^{2}(t+$ $1)^{2}(t-i)$

17. II $421^{3} \quad y^{2}=x^{3}+t \in x^{2}+t\left(2 t^{3}+1\right) x+\frac{t}{\epsilon}\left(-t^{5}-(1+i) t^{4}-t^{3}+(1-\right.$ i) $\left.\mathrm{t}^{2}+\mathrm{t}-1\right)$

$-\Delta=t^{12}+(2-i) t^{10}+(2+2 i) t^{9}+i t^{8}+(1+i) t^{7}+2 t^{6}+(1+i) t^{5}+(1+$ i) $t^{4}+2 t^{3}=t^{3}(t+(1+i))^{4}(t+i)^{2}(t-(1+i))(t-(1-i))(t+1)$, where $\epsilon^{2}=2-i$

18. II $4 \mathbf{1}^{5} \quad y^{2}=x^{3}+t \in x^{2}+t\left(2 t^{3}+1+i\right) x+\frac{t}{\epsilon}\left((1-2 i) t^{5}-i t^{4}+t^{2}+(2-i) t-2 i\right)$ $-\Delta=t^{12}+i t^{10}+(1-i) t^{9}+2 t^{8}+(2+i) t^{7^{6}}-i t^{6}+(2+i) t^{5}+2 t^{4}+(2+i) t^{3}=$ $t^{3}(t-(2-i))^{4}(t-1)(t+i)(t-i)(t+1)(t-(1+i))$, where $\epsilon^{2}=i$

19. II $3^{3} \quad y^{2}=x^{3}+t^{2} x^{2}+t(t+1) x+t\left(2+2 t^{2}+t\right)$

$-\Delta=t^{9}+2 t^{3}=t^{3}(t-1)^{3}(t+1)^{3}$

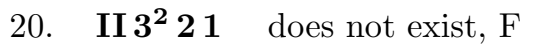

21. II $3^{2} \mathbf{1}^{3} \quad y^{2}=x^{3}+t(t+1) x^{2}+t(2 t+1) x+t\left(2+2 t^{2}+2 t\right)$

$-\Delta=t^{9}+2 t^{8}+t^{7}+t^{5}+2 t^{4}+2 t^{3}=-t^{3}(t-1)^{3}\left(-t^{3}+t^{2}-t-1\right)$

22. II $32^{3} \quad y^{2}=x^{3}+t \in x^{2}+t\left(2 t^{3}+i\right) x+\frac{t}{\epsilon}\left((1-i) t^{5}-i t^{4}-2 i t^{2}-i t-i\right)$ $-\Delta=t^{12}+i t^{10}+(2+2 i) t^{9}+2 t^{8}+2 t^{7^{6}}+t^{6}+2 t^{5}+(2-i) t^{4}-2 i t^{3}=$ $t^{3}(t+1)^{3}(t-(2-i))^{2}(t-1)^{2}(t-i)^{2}$, where $\epsilon^{2}=i$

23. II $32^{2} 1^{2} \quad y^{2}=x^{3}+t(t+1) x^{2}+t(t+2) x+t\left((2+i) t^{2}+(2+i) t+2+i\right)$ $-\Delta=(1-i) t^{9}-(1+i) t^{8}+(1-i) t^{7}+(1-i) t^{6}+(1-i) t^{5}-(1+i) t^{4}+t^{3}=$ $i\left(t-\frac{1}{1-i}\right)^{2}\left(2 t^{2}+2 i t^{2}+1\right) t^{3}(t-1)^{2}$

24. II $321^{4} \quad y^{2}=x^{3}+t^{2} x^{2}+t(t+1) x+t\left(1+t^{2}\right)$

$-\Delta=2 t^{9}+t^{8}+t^{7}+2 t^{3}=t^{3}(t-1)^{2}\left(-t^{4}-t^{3}+t-1\right)$

25. II $31^{6} \quad y^{2}=x^{3}+\left(t^{2}+i t\right) x^{2}+\left(i t^{2}+2 t+t^{2}\right) x+2 t^{3}+2 t^{2}-i t^{2}+i t$

$-\Delta=t^{9}+t^{8}+t^{6}+t^{4}+t^{3}=t^{3}\left(t^{6}+t^{5}+t^{3}+t+1\right)$

26. II $2^{4} 1 \quad y^{2}=x^{3}+t^{2} x^{2}+2 t x+t\left(2 t^{5}+2 t^{4}+1+t^{2}+2 t\right)$

$-\Delta=t^{12}+t^{11}+2 t^{9}+t^{8}+2 t^{7}+t^{6}+t^{3}=t^{3}(t-1)^{2}\left(t^{3}+t^{2}+t+2\right)^{2}(t-2)$

27. II $2^{3} \mathbf{1}^{3} \quad y^{2}=x^{3}+i t x^{2}+t\left(2 t^{3}+2+i\right) x+t\left((1+2 i) t^{5}+2+(2+i) t^{3}+\right.$ $\left.2 t^{2}+2 i t\right)$

$-\Delta=t^{12}+2 t^{10}+(1+i) t^{9}+i t^{7}-i t^{6}+t^{5}+i t^{4}+(1+i) t^{3}=t^{3}(t-(1+$ $i))^{2}(t+(1+i))^{2}(t+1)^{2}(t-1)(t-i)(t-(1-i))$ 
28. II $2^{2} 1^{5} \quad y^{2}=x^{3}+t \in x^{2}+t\left(2 t^{3}+2 i\right) x+\frac{t}{\epsilon}\left((1-i) t^{5}-i t^{4}+t^{3}+t^{2}+\right.$ $(-1+i) t+(1-i))$

$-\Delta=t^{12}+(1+i) t^{10}+t^{9}+(2+i) t^{8}+i t^{7}+(2+2 i) t^{6}+2 t^{5}+2 i t^{4}+$ $2 i t^{3} t^{3}(t-(1+i))^{2}(t-1)^{2}(t+1)(t+i)(t-i)(t+(1+i))(t-(1-i))$, where $\epsilon^{2}=1+i$

29. II $2 \mathbf{1}^{7} \quad y^{2}=x^{3}+t \in x^{2}+t\left(2 t^{3}+2+i\right) x+\frac{t}{\epsilon}\left((-1+2 i) t^{5}+i t^{4}+(-2+\right.$ $\left.2 i) t^{3}+t^{2}+(-2+i) t+i\right)$

$-\Delta=t^{12}-i t^{10}+(4+2 i) t^{9}+2 t^{8}+(5+5 i) t^{7}-2 i t^{6}+(2+4 i) t^{5}+(4+4 i) t^{3}=$ $t^{3}(t-(-1-i))^{2}(t-1)(t+1)(t-i)(t-(-1-i))(t+i)(t-(-1+i))(t-(1-i))$, where $\epsilon^{2}=-i$

30. II $1^{9} \quad y^{2}=x^{3}+t(t+1) x^{2}+2 t x+t\left(t^{4}+2 t^{3}+1\right)$

$-\Delta=2 t^{11}+t^{10}+2 t^{8}+t^{6}+2 t^{5}+t^{3}=-t^{3}(t-1)\left(1+t+t^{3}+t^{4}+t^{7}\right)$

31. II II II II $\mathrm{y}^{2}=\mathrm{x}^{3}-\mathrm{t}\left(\mathrm{t}^{2}-1\right) \mathrm{x}+\mathrm{t}\left(\mathrm{t}^{2}-1\right)\left(\mathrm{t}^{2}-\mathrm{t}-1\right)$

$-\Delta=t^{9}-t^{3}=\left(t\left(t^{2}-1\right)\right)^{3}$

32. IIII 6 $\mathrm{y}^{2}=\mathrm{x}^{3}+\mathrm{t} \mathrm{x}^{2}+\mathrm{t}\left(2 \mathrm{t}^{2}+\mathrm{t}+2\right) \mathrm{x}+\mathrm{t}\left(\mathrm{t}^{4}+\mathrm{t}^{3}+\mathrm{t}^{2}+\mathrm{t}+1\right)$

$-\Delta=t^{9}+t^{6}+t^{3}=t^{3}(t-1)^{6}$

33. IIII5 $1 \quad y^{2}=x^{3}+t x^{2} i+t\left(t^{2}+t+2\right) x+t^{5} i$

$-\Delta=2 t^{9}+t^{8}+t^{7}+2 t^{5}+2 t^{4}+t^{3}=-t^{3}(t-1)^{5}(t+1)$

34. IIII $42 \quad y^{2}=x^{3}+t x^{2} i+t\left(2 t^{2}+t+2\right) x+t\left(t^{4} i+t^{2} i+i\right)$

$-\Delta=t^{9}+t^{8}+2 t^{7}+t^{6}+2 t^{5}+t^{4}+t^{3}=t^{3}(t-1)^{4}(t+1)^{2}$

35. IIII $4 \mathbf{1}^{2} \quad y^{2}=x^{3}+t x^{2}+t\left(2 t^{2}+t+2\right) x+t\left(2 t^{4}+t^{2}+2\right)$

$-\Delta=t^{9}+2 t^{8}+t^{7}+t^{6}+t^{5}+2 t^{4}+t^{3}=t^{3}(t-1)^{4}\left(t-\frac{1}{i}\right)\left(t+\frac{1}{i}\right)$

36. IIII $3^{2} \quad y^{2}=x^{3}+t x^{2}+t\left(t^{2}+t+2\right) x+t\left(t^{4}+2 t^{3}+t^{2}+1\right)$

$-\Delta=2 t^{9}+t^{3}=-t^{3}(t-1)^{3}(t+1)^{3}$

37. IIII $321 \quad y^{2}=x^{3}+t x^{2}+t\left(t^{2}+t+2\right) x+t\left((2-i) t^{4}-t^{3} i+(1+i) t^{2}-t i+2 i\right)$ $-\Delta=2 t^{9}+(2+i) t^{8}+(2+i) t^{7}+-i t^{6}+(1+i) t^{5}+(1+i) t^{4}+t^{3}=$ $-t^{3}\left(t-\frac{1}{i}\right)^{3}(t-1)^{2}\left(t+\frac{1}{i}\right)$

38. IIII $31^{3} \quad y^{2}=x^{3}+t x^{2}+t\left(t^{2}+1\right) x+t\left(t^{4}+2 t^{3}+2\right)$

$-\Delta=2 t^{9}+t^{7}+2 t^{6}+2 t^{4}+2 t^{3}=-t^{3}(t-1)^{3}\left(t^{3}+2 t+2\right)$

39. IIIII $2^{3} \quad y^{2}=x^{3}+t x^{2} \mathfrak{i}+t\left(2 t^{2}+t+2\right) x+t\left(t^{4} i-t^{3} i-t i+i\right)$

$-\Delta=t^{9}+t^{8}+2 t^{6}+t^{4}+t^{3}=t^{3}(t-1)^{2}\left(t-\frac{1}{i}\right)^{2}\left(t+\frac{1}{i}\right)^{2}$

40. IIII $2^{2} 1^{2} \quad y^{2}=x^{3}+t x^{2}+t\left(2 t^{2}+t+2\right) x+t\left(t^{4}+2 t^{3}+2 t^{2}+2 t+1\right)$

$-\Delta=t^{9}+2 t^{7}+2 t^{5}+t^{3}=t^{3}(t-1)^{2}(t+1)^{2}\left(t-\frac{1}{i}\right)\left(t+\frac{1}{i}\right)$

41. IIII $21^{4} \quad y^{2}=x^{3}+t x^{2}+t\left(2 t^{2}+t+2\right) x+t\left(t^{4}+t^{3}+2 t^{2}+2 t+2\right)$

$-\Delta=t^{9}+2 t^{5}+2 t^{4}+t^{3}=t^{3}(t-1)^{2}\left(t^{4}-t^{3}+t+1\right)$

42. II II 1 $1^{6} \quad y^{2}=x^{3}+t x^{2}+t\left(t^{2}+t+2\right) x+t\left(t^{4}+t^{3}+t^{2}+2 t+1\right)$

$-\Delta=2 t^{9}+2 t^{7}+2 t^{5}+t^{3}=-t^{3}(t-1)^{2}(t+1)\left(t^{4}+1\right)$ 


\subsubsection{Case 1B}

$$
\begin{gathered}
\delta=4, \quad r=0 \quad \text { Type II } \\
y^{2}=x^{3}+t c_{1} x^{2}+t^{2} c_{2} x+t c_{5}, \quad t \nmid c_{5}, t \nmid c_{1} \\
-\Delta=t^{2} c_{1}^{2}\left(t^{2} c_{1} c_{5}-t^{4} c_{2}^{2}\right)+t^{6} c_{2}^{3}
\end{gathered}
$$

1. II 8 does not exist, $\mathbf{F}$

2. II 7 1 $y^{2}=x^{3}+t(t+1) x^{2}+t^{2} x+t(2+t)$

$-\Delta=2 t^{5}+t^{4}=-t^{4}(t-1)$

3. II 62 does not exist, [F]

4. II $6 \mathbf{1}^{2} \quad y^{2}=x^{3}+t(t+1) x^{2}+2 t^{2} x+t(2+t)$

$-\Delta=2 t^{6}+2 t^{5}+t^{4}=t^{4}\left(-t^{2}-t+1\right)$

5. II 53 does not exist, $\mathbb{E}$

6. II $521 y^{2}=x^{3}+t(t+i) x^{2}+(1-i) t^{2} x+t(2+t \mathfrak{i})$

$-\Delta=2 t^{7}+(-1+i) t^{6}+2 t^{5}-i t^{4}=t^{4}\left(-i-t-(1-i) t^{2}-t^{3}\right)$

7. II $51^{3} \quad y^{2}=x^{3}+t(t+1) x^{2}+t^{2} x+t(t+1)$

$-\Delta=t^{7}+2 t^{5}+2 t^{4}=t^{4}\left(2-t+t^{3}\right)$

8. II $4^{2} \quad y^{2}=x^{3}+t(t+1) x^{2}+t^{2} x+t(1+2 t)$

$-\Delta=2 t^{8}+t^{7}+t^{5}+2 t^{4}=-t^{4}(t-1)^{4}$

9. II $431 \quad y^{2}=x^{3}+t(t+1) x^{2}+t^{2} x+t((2-i) t+1+i)$

$-\Delta=(-1+i) t^{8}-(2+i) t^{7}+(-2+i) t^{5}-(1+i) t^{4}=-(1-i) t^{4}(t-1)\left(t-\frac{1}{i}\right)^{3}$

10. II $42^{2} \quad y^{2}=x^{3}+t(t+i) x^{2}+t^{2} x-i t$

$-\Delta=t^{8}+t^{6}+t^{4}=t^{4}(t-1)^{2}(t+1)^{2}$

11. II $421^{2} \quad y^{2}=x^{3}+t(t+1) x^{2}+t^{2} x i+t(t i+1+i)$

$-\Delta=(2+2 i) t^{8}-i t^{7}+(2+i) t^{6}-i t^{5}+(2+2 i) t^{4}=t^{4}(t-1)^{2}\left((2+2 i) t^{2}+\right.$ $\mathrm{t}+(2+2 i))$

12. II $41^{4} \quad y^{2}=x^{3}+t(t+1) x^{2}+2 t^{2} x+t$

$-\Delta=t^{8}+t^{7}+2 t^{6}+2 t^{4}=-t^{4}\left(2-t+t^{2}-t^{3}\right)(t-1)$

13. II $3^{2} \mathbf{2}$ does not exist, $\mathbb{F}$

14. II $3^{2} \mathbf{1}^{2} \quad y^{2}=x^{3}+t(t+1) x^{2}+t^{2} x i+t\left(2+(1-i) t^{2}+t\right)$

$-\Delta=(-1+i) t^{9}+t^{8}+2 t^{7}+(1-i) t^{6}+2 t^{5}+t^{4}=-t^{4}(t-1)^{3}\left((1-i) t^{2}-t+1\right)$

15. II $32^{2} 1 \quad y^{2}=x^{3}+t(t+i) x^{2}+2 t^{2} x+t\left(1-(1+i) t^{2}+(1+i) t\right)$

$-\Delta=(1+i) t^{9}+2 i t^{8}-(1+i) t^{7}+(1-i) t^{6}+(2+i) t^{5}+t^{4} i=i t^{4}(t-$ $1)^{2}\left(t-\frac{1}{1-1}\right)^{2}((1-i) t+i)$

16. II $321^{3} \quad y^{2}=x^{3}+t(t+1) x^{2}+t^{2} x+t\left(2+t^{2}\right)$

$-\Delta=2 t^{9}+t^{8}+2 t^{6}+t^{4}=t^{4}(t-1)^{2}\left(1-t-t^{2}-t^{3}\right)$ 
17. II $31^{5} \quad y^{2}=x^{3}+t(t+i) x^{2}+t^{2} x+t\left(1+(2-\mathfrak{i}) t^{2}\right)$

$-\Delta=(-2+i) t^{9}+t^{8}+(2-i) t^{7}-(1+i) t^{6}+t^{4} i=-t^{4}(t-1)(t+1)(i-$ $\left.\mathrm{t}^{2}+(2-i) \mathrm{t}^{3}\right)$

18. II $2^{4} \quad y^{2}=x^{3}+t(t+\epsilon) x^{2}+t^{2} x+t\left(-i t^{3}+t^{2}(\epsilon+\epsilon i)+(1-i) t+2 i \epsilon\right)$

$-\Delta=t^{10} i+(2 \epsilon-i \epsilon) t^{9}+i t^{8}+(i \epsilon+\epsilon) t^{7}+2 i t^{6}+(2 \epsilon-i \epsilon) t^{5}+2 i t^{4}=$ $i t^{4}\left(t-\frac{1}{i}\right)^{2}\left(t+\frac{1}{i}\right)^{2}\left(t-\frac{\epsilon}{1+i}\right)^{2}$, where $\epsilon^{4}=-1$

19. II $2^{3} 1^{2} \quad y^{2}=x^{3}+t(t+1) x^{2}+t^{2} x+t\left(2 t^{3}+(1+i) t^{2}+t i+i\right)$

$-\Delta=t^{10}+(-1+2 i) t^{9}-(2+i) t^{8}-i t^{7}+(2+2 i) t^{6}+2 i t^{5}-i t^{4}=$ $-t^{4}(t-1)^{2}\left(t-\frac{1}{i}\right)^{2}\left(-t^{2}-t-i\right)$

20. II $2^{2} 1^{4} \quad y^{2}=x^{3}+t(t+1) x^{2}+2 t^{2} x+t\left(t^{3}+2 t^{2}+1\right)$

$-\Delta=2 t^{10}+t^{9}+t^{8}+2 t^{4}=t^{4}(t-1)^{2}\left(2+t-t^{3}-t^{4}\right)$

21. II $21^{6} \quad y^{2}=x^{3}+t(t+1) x^{2}+t^{2} x+t\left(2+2 t^{3}+2 t^{2}\right)$

$-\Delta=\mathrm{t}^{10}+\mathrm{t}^{9}+\mathrm{t}^{8}+\mathrm{t}^{7}+\mathrm{t}^{6}+\mathrm{t}^{4}=-\mathrm{t}^{4}(\mathrm{t}-1)\left(\mathrm{t}-\frac{1}{\mathrm{i}}\right)\left(\mathrm{t}+\frac{1}{\mathrm{i}}\right)\left(1+\mathrm{t}+\mathrm{t}^{2}-\mathrm{t}^{3}\right)$

22. II $1^{8} \quad y^{2}=x^{3}+t(t+1) x^{2}+t^{2} x+t\left(t^{4} i-i t^{3}+(1+i) t^{2}+(1-i) t+1\right)$ $-\Delta=-i t^{11}+t^{10} i-(1+i) t^{9}+(-2+i) t^{7}+(2-i) t^{6}+(-1+i) t^{5}+2 t^{4}=$ $-t^{4}(t-1)(t+i)(t-i)\left(2+(1+i) t+t^{2}+i t^{4}\right)$

23. IIII 5 $y^{2}=x^{3}+t x^{2}+t^{3} x+t\left(2 t^{4}+t^{3}+2 t^{2}+2 t^{2}+2\right)$

$-\Delta=2 t^{9}+2 t^{8}+2 t^{7}+t^{6}+t^{5}+t^{4}=-t^{4}(t-1)^{5}$

24. IIII $41 \quad y^{2}=x^{3}+t x^{2}+t^{2}(2 t+1) x+t\left(t^{4}+2 t^{3}+t^{2}+2\right)$

$-\Delta=t^{9}+2 t^{7}+2 t^{6}+t^{4}=t^{4}(t-1)^{4}(t+1)$

25. II II $32 \quad y^{2}=x^{3}+t x^{2} i+t^{3} x+t\left(t^{4} i+t^{3} i-t^{2} i+t i+2 i\right)$

$-\Delta=2 t^{9}+t^{8}+2 t^{7}+t^{6}+2 t^{5}+t^{4}=-t^{4}(t-1)^{3}(t+1)^{2}$

26. IIII $31^{2} \quad y^{2}=x^{3}+t x^{2}+t^{3} x+t\left(t^{4}+t^{3}+2 t^{2}+2\right)$

$-\Delta=2 t^{9}+2 t^{7}+t^{6}+t^{4}=-t^{4}(t-1)^{3}\left(t-\frac{1}{i}\right)\left(t+\frac{1}{i}\right)$

27. IIII $2^{2} 1 \quad y^{2}=x^{3}+t x^{2} i+t^{3} x+t\left(t^{4} i-t^{3}+t^{2} i+t i+2 i\right)$

$-\Delta=2 t^{9}+t^{8}+t^{7}+2 t^{6}+2 t^{5}+t^{4}=-t^{4}\left(t-\frac{1}{i}\right)^{2}\left(t+\frac{1}{i}\right)^{2}(t-1)$

28. IIII $21^{3} \quad y^{2}=x^{3}+t x^{2}+t^{2}(t+1) x+t\left(2 t^{4}+t^{3}+2\right)$

$-\Delta=2 t^{9}+2 t^{8}+t^{7}+t^{5}=t^{4}(t-1)^{2}\left(2 t^{3}+2 t+1\right)$

29. IIIII $1^{5} \quad y^{2}=x^{3}+t x^{2}+t^{3} x+t\left(t^{4}+t^{3}+t^{2}+t+2\right)$

$-\Delta=2 t^{9}+2 t^{7}+2 t^{6}+2 t^{5}+t^{4}=-t^{4}(t-1)(t+1)\left(t^{3}-t+1\right)$

30. IIIII 5 $y^{2}=x^{3}+t x^{2} i+t^{3} x+t\left(t^{3} i-t^{2} i-t i+2 i\right)$

$-\Delta=2 t^{9}+2 t^{8}+2 t^{7}+t^{6}+t^{5}+t^{4}=-t^{4}(t-1)^{5}$

31. II III $41 \quad y^{2}=x^{3}+t \in x^{2}-t^{3} x i+\frac{t}{\epsilon}\left((2+2 i) t^{3}+(2+i) t^{2}+t+2+2 i\right)$

$-\Delta=-t^{9} i+(1-i) t^{8}+t^{7}+(3-i) t^{6}+(1-i) t^{5}+t^{4}=-t^{4} i(t+1)^{4}\left(t-\frac{1}{i}\right)$

32. IIIII $32 \mathrm{y}^{2}=\mathrm{x}^{3}+\mathrm{t} \mathrm{x}^{2}+\mathrm{t}^{3} \mathrm{x}+\mathrm{t}\left(\mathrm{t}^{3}+2 \mathrm{t}^{2}+\mathrm{t}+2\right)$

$-\Delta=2 t^{9}+t^{8}+2 t^{7}+t^{6}+2 t^{5}+t^{4}=-t^{4}(t-1)^{3}(t+1)^{2}$ 
33. IIIII 3 $1^{2} \quad y^{2}=x^{3}+t x^{2}+t^{2}(2 t+1) x+t\left(2 t^{3}+t^{2}+t+2\right)$

$-\Delta=t^{9}+t^{8}+2 t^{7}+2 t^{6}+2 t^{5}+t^{4}=-t^{4}(t-1)^{3}\left(2 t^{2}+2 t+1\right)$

34. IIIII 2 $2^{2} 1 \quad y^{2}=x^{3}+t x^{2}+t^{3} x+t\left(2 t^{3}+t^{2}+t+2\right)$

$-\Delta=2 t^{9}+t^{8}+t^{7}+2 t^{6}+2 t^{5}+t^{4}=-t^{4}(t-1)\left(t-\frac{1}{i}\right)^{2}\left(t+\frac{1}{i}\right)^{2}$

35. II III $21^{3} \quad y^{2}=x^{3}+t x^{2}+t^{2}(2 t+1) x+t\left(t^{3}+2 t^{2}+2 t+1\right)$

$-\Delta=t^{9}+t^{8}+t^{6}+t^{5}+2 t^{4}=t^{4}(t-1)^{2}\left(t^{3}+2 t+2\right)$

36. IIIII $1^{5} \quad y^{2}=x^{3}+t^{2}+t^{2}(t+1) x+t\left(t^{3}+2 t^{2}+2\right)$

$-\Delta=2 t^{9}+t^{8}+t^{7}+t^{6}+t^{4}=-t^{4}(t-1)(t+1)\left(t^{3}+2 t^{2}+1\right)$

\subsubsection{Case $1 \mathrm{C}$}

$$
\begin{gathered}
\delta=6, \quad r=0 \quad \text { Type II } \\
y^{2}=x^{3}+t^{2} c_{0} x^{2}+t^{2} c_{2} x+t c_{5}, \quad t \nmid c_{5}, t \nmid c_{2} \\
-\Delta=t^{4} c_{0}^{2}\left(t^{3} c_{0} c_{5}-t^{4} c_{2}^{2}\right)+t^{6} c_{2}^{3}
\end{gathered}
$$

1. II 6 does not exist, G

2. II $51 \mathrm{y}^{2}=\mathrm{x}^{3}+\mathrm{t}^{2} \mathrm{x}^{2}+\mathrm{t}^{2}\left(\mathrm{t}^{3}+\mathrm{t}+1\right) \mathrm{x}+\mathrm{t}\left(2 \mathrm{t}^{5}+\mathrm{t}^{3}+\mathrm{t}^{2}+2\right)$

$-\Delta=2 t^{12}+t^{11}+t^{10}+2 t^{8}+2 t^{7}+t^{6}=-t^{6}(t-1)^{5}(t+1)$

3. II $42 \quad y^{2}=x^{3}+t^{2} x^{2}+t^{2}\left(t^{2}+t+2\right) x+t\left(2 t^{5}+t^{4}+2+2 t^{2}+2 t\right)$

$-\Delta=t^{4}\left(t^{3}\left(2 t^{5}+t^{4}+2 t^{2}+2 t+2\right)+2 t^{4}\left(t^{2}+t+2\right)^{2}+t^{6}\left(t^{2}+t+2\right)^{3}=\right.$ $-t^{6}(t-1)^{4}(t+1)^{2}$

4. II $4 \mathbf{1}^{2} \quad y^{2}=x^{3}+t^{2} x^{2}+t^{2}\left(t^{2}+t+2\right) x+t\left(2 t^{5}+t^{3}+2 t^{2}+1\right)$

$-\Delta=2 t^{12}+t^{11}+2 t^{10}+2 t^{9}+2 t^{8}+t^{7}+2 t^{6}=-t^{6}(t-1)^{4}(t-i)(t+i)$

5. $\quad$ II $^{2}{ }^{2}$ does not exist, $\mathrm{G}$

6. II $321 \quad y^{2}=x^{3}+t^{2} x^{2}+\left(2 t^{4}+t^{3}+t^{2}\right) x+i t^{5}+t^{4}+2 t^{3}+2(1+i) t^{2}+t$ $-\Delta=t^{12}+(2+i) t^{11}+2 t^{10}+t^{9}+(1+2 i) t^{8}+t^{7}+t^{6}=-t^{6}(t-1)^{3}\left(-t^{3}+\right.$ $\left.(1-i) t^{2}+t+1\right)$

7. II $31^{3} \quad y^{2}=x^{3}+t^{2} x^{2}+t^{2}\left(1+t^{2}+t\right) x+t\left(t^{5}+2 t^{4}+2 t^{3}+2 t^{2}+t+1\right)$ $-\Delta=t^{12}+2 t^{10}+t^{9}+t^{7}+t^{6}=-t^{6}(t-1)^{3}\left(2 t^{3}+t+1\right)$

8. II $2^{3} \quad y^{2}=x^{3}+2 t^{2} x^{2}+\left(t^{4}+t^{3}+\phi_{1} t^{2}\right) x+t^{2} \phi_{1}^{3}+2 t^{5}+\left(2+\phi_{1}\right) t^{4}+$ $\left(2+\phi_{1}\right) \mathrm{t}^{3}+2 \phi_{1}^{2} \mathrm{t}^{2}+\mathrm{t}$

$-\Delta=2 t^{12} \phi_{1}^{3}+2 t^{11}+2 t^{9}+2 t^{7}+t^{6} \phi_{1}^{3}=-t^{6}(t-1)^{2}(t+1)^{2}\left(2 t^{2} \phi_{1}^{3}+2 t+\phi_{1}^{3}\right)$, where $\phi_{1}^{6}=-1$

9. II $2^{2} \mathbf{1}^{2} \quad y^{2}=x^{3}+t^{2} x^{2}+\left(t^{4}+t^{3}+2 t^{2}\right) x+t^{6}+2 t^{4}+t^{3}+t^{2}+t$

$-\Delta=t^{12}+t^{11}+t^{9}+t^{7}+2 t^{6}=-t^{6}(t-1)^{2}(t+1)^{2}\left(2 t^{2}+2 t+1\right)$

10. II 2 1 $1^{4} y^{2}=x^{3}+t^{2} x^{2}+\left(t^{4}+t^{3}+t^{2}\right) x+t^{6}+t^{5}+t^{4}+t^{3}+t^{2}+t$ $-\Delta=t^{12}+2 t^{11}+t^{10}+t^{7}+t^{6}=-t^{6}(t-1)^{2}(t+1)\left(2 t^{3}+t+2\right)$ 
11. II $1^{6} \quad y^{2}=x^{3}+t^{2} x^{2}+t^{2}\left(t^{2}+t+1\right) x+t\left(2 t^{5}+t^{3}+2 t^{2}+1\right)$

$-\Delta=t^{4}\left(t^{3}\left(2 t^{5}+t^{3}+2 t^{2}+1\right)+2 t^{4}\left(t^{2}+t+1\right)^{2}\right)+t^{6}\left(t^{2}+t+1\right)^{3}=$ $-t^{6}(t-1)(t+1)\left(t^{4}+2 t^{3}+t+1\right)$

12. II II $\mathrm{y}^{2}=\mathrm{x}^{3}+\mathrm{t}^{2} \mathrm{x}+\mathrm{t}\left(\mathrm{t}^{4}+\mathrm{t}^{3}+\mathrm{t}^{2}+\mathrm{t}+1\right)$

$-\Delta=2 t^{6}$

13. II II II $y^{2}=x^{3}+t^{2}(2 t+1) x+t\left(t^{4}+t^{3}+t^{2}+2 t+1\right)$

$-\Delta=t^{9}+2 t^{6}=2 t^{6}(2 t+1)^{3}$

14. II II III $y^{2}=x^{3}+t^{2}(2 t+1) x+t\left(t^{4}+t^{3}+2 t^{2}+t+1\right)$

$-\Delta=t^{9}+2 t^{6}=2 t^{6}(2 t+1)^{3}$

15. II IIIIII $y^{2}=x^{3}+t^{2}(2 t+1) x+t\left(t^{2}+t+1\right)$

$-\Delta=t^{9}+2 t^{6}=2 t^{6}(2 t+1)^{3}$

\subsubsection{Case 1D}

$$
\begin{gathered}
\delta=7, \quad r=0 \quad \text { Type II } \\
y^{2}=x^{3}+t^{2} c_{0} x^{2}+t^{3} c_{1} x+t c_{5}, \quad t \nmid c_{5}, t \nmid c_{0} \\
-\Delta=t^{4} c_{0}^{2}\left(t^{3} c_{0} c_{5}-t^{6} c_{1}^{2}\right)+t^{9} c_{1}^{3}
\end{gathered}
$$

1. II $5 \mathrm{y}^{2}=\mathrm{x}^{3}+\mathrm{t}^{2} \mathrm{x}^{2}+\mathrm{t}^{2}(\mathrm{t}+1) \mathrm{x}+\mathrm{t}\left(2 \mathrm{t}^{5}+\mathrm{t}^{4}+\mathrm{t}+1\right)$

$-\Delta=t^{4}\left(t^{3}\left(2 t^{5}+t^{4}+t+1\right)+2 t^{6}(t+1)^{2}\right)+t^{9}(t+1)^{3}=-t^{7}(t-1)^{5}$

2. II $41 \mathrm{y}^{2}=\mathrm{x}^{3}+\mathrm{t}^{2} \mathrm{x}^{2}+\mathrm{t}^{2}(\mathrm{t}+1) \mathrm{x}+\mathrm{t}\left(2 \mathrm{t}^{5}+\mathrm{t}^{4}+2 \mathrm{t}^{3}+2\right)$

$-\Delta=2 t^{12}+t^{10}+t^{9}+2 t^{7}=-t^{7}(t+1)(t-1)^{4}$

3. II $32 y^{2}=x^{3}+t^{2} x^{2}+t^{2}(t+1) x+t\left(2 t^{5}+2 t+1\right)$

$-\Delta=2 t^{12}+t^{11}+2 t^{10}+t^{9}+2 t^{8}+t^{7}=-t^{7}(t-1)^{3}(t+1)^{2}$

4. II $3 \mathbf{1}^{2} \quad y^{2}=x^{3}+2 t^{2} x^{2}+\left(t^{3}+t^{2}\right) x+t^{6}+t^{4}+t^{2}+t$

$-\Delta=2 t^{12}+t^{11}+t^{10}+t^{9}+2 t^{8}+2 t^{7}=-t^{7}(t-1)^{3}\left(t^{2}+2 t+2\right)$

5. II $21^{3} \quad y^{2}=x^{3}+2 t^{2} x^{2}+t^{2}(t+1) x+t\left(2 t^{5}+t^{2}+1\right)$

$-\Delta=t^{4}\left(2 t^{3}\left(2 t^{5}+t^{2}+1\right)+2 t^{6}(t+1)^{2}\right)+t^{9}(t+1)^{3}=-t^{7}(t-1)^{2}(t+$ 1) $\left(2 t^{2}+t+1\right)$

6. II $2^{2} 1 \quad y^{2}=x^{3}+t^{2} x^{2}+t^{2}(t+1) x+t\left(2 t^{5}+2 t^{3}+t^{2}+2 t+1\right)$

$-\Delta=2 t^{12}+t^{11}+t^{10}+2 t^{9}+2 t^{8}+t^{7}=-t^{7}(t-i)^{2}(t+i)^{2}(t-1)$

7. II $1^{5} \quad y^{2}=x^{3}+t^{2} x^{2}+t^{2}(t+1) x+t\left(t^{5}+2 t^{4}+2 t^{3}+t^{2}+t+1\right)$

$-\Delta=t^{4}\left(t^{3}\left(t^{5}+2 t^{4}+2 t^{3}+t^{2}+t+1\right)+2 t^{6}(t+1)^{2}\right)+t^{9}(t+1)^{3}=$ $-t^{7}(t-1)(t+1)\left(2 t^{3}+t+1\right)$

8. IIIV does not exist, $\mathbf{E}$

9. IIII 1 does not exist, E

10. IIII 2 does not exist, $\mathbb{E}$ 
11. IIII $\mathbf{1}^{2}$ does not exist, $\mathrm{E}$

12. IIIII 2 does not exist, $\mathrm{E}$

13. II III $\mathbf{1}^{2}$ does not exist, $\mathbb{E}$

\subsubsection{Case 1E}

$$
\begin{gathered}
\delta=9, \quad r=0 \quad \text { Type II } \\
y^{2}=x^{3}+t^{3} c_{1} x+t c_{5}, \quad t \nmid c_{5}, t \nmid c_{1} \\
-\Delta=t^{9} c_{1}^{3}
\end{gathered}
$$

1. II 3 does not exist,

2. II 21 does not exist, $\mathbb{E}$

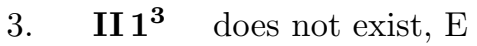

4. IIII $y^{2}=x^{3}+t^{3}(2 t+1) x+t\left(t^{3}+t+1\right)$

$-\Delta=\mathrm{t}^{9}(2 \mathrm{t}+1)^{3}$

5. II III $y^{2}=x^{3}+t^{3}(2 t+1) x+t\left(t^{2}+t+1\right)$ $-\Delta=t^{9}(2 t+1)^{3}$

\subsubsection{Case $1 \mathrm{~F}$}

$$
\begin{gathered}
\delta=12, \quad r=0 \quad \text { Type II } \\
y^{2}=x^{3}+t^{4} c_{0} x+t c_{5}, \quad t \nmid c_{5}, t \nmid c_{0} \\
-\Delta=t^{12} c_{0}^{3}
\end{gathered}
$$

\subsubsection{Case 2}

$$
\begin{gathered}
\delta=3, \quad r=1 \quad \text { Type III } \\
y^{2}=x^{3}+t c_{1} x^{2}+t c_{3} x+t^{2} c_{4}, \quad t \nmid c_{3} \\
-\Delta=t^{2} c_{1}^{2}\left(t^{3} c_{1} c_{4}-t^{2} c_{3}^{2}\right)+t^{3} c_{3}^{3}
\end{gathered}
$$

1. III III III III $y^{2}=x^{3}-t(t-1)\left(t-\left(\alpha+\alpha^{3}\right)\right) x+\left(\alpha^{5}-\alpha^{3}\right)\left(t^{2}(t-1)^{2}\right)$ $-\Delta=t^{9}-t^{6}\left(1+\left(\alpha^{3}-\alpha\right)\right)+\left(\alpha^{3}-\alpha\right) t^{3}=t^{3}(t-1)^{3}\left(t-\left(\alpha+\alpha^{3}\right)\right)^{3}$, where $\alpha^{4}+\alpha^{2}=1$.

2. III III III II $y^{2}=x^{3}-t\left(t^{2}-1\right) x+t^{2}(t-1)^{2}$

$-\Delta=\mathrm{t}^{9}+\mathrm{t}^{3}=\mathrm{t}^{3}\left(\mathrm{t}^{2}+1\right)^{3}$

3. III III II II $y^{2}=x^{3}-t\left(t^{2}+1\right) x+t^{2}\left(t^{2}+1\right)^{2}$ $-\Delta=t^{9}+t^{3}=t^{3}\left(t^{2}+1\right)^{3}$ 
4. III II II II $y^{2}=x^{3}-t\left(t^{2}+1\right) x+t^{2}\left(t^{2}+1\right)(t-1)$

$-\Delta=\mathrm{t}^{9}+\mathrm{t}^{3}=\mathrm{t}^{3}\left(\mathrm{t}^{2}+1\right)^{3}$

5. IIIII 6 does not exist, $G$

6. IIIII $51 y^{2}=x^{3}+t x^{2} i+t\left(t^{2}+2\right) x+t^{2}\left(t^{3} i-t^{2} i-t i+i\right)$

$-\Delta=2 t^{9}+t^{8}+t^{7}+2 t^{5}+2 t^{4}+t^{3}$

7. III II $42 y^{2}=x^{3}+t x^{2} i+t\left(t^{2}+1\right) x+t^{2}\left(t^{3}+t^{2} i+(1-i) t+2+2 i\right)$

$-\Delta=2 t^{9}-(1-i) t^{8}-t^{7}+(1-i) t^{6}+(1-i) t^{5}+t^{4}+t^{3} i$

8. III II 4 $1^{2} \quad y^{2}=x^{3}+t x^{2}+t\left(2 t^{2}+1\right) x+t^{2}\left(2 t^{3}+t^{2}+t+2\right)$

$-\Delta=t^{9}+2 t^{8}+2 t^{7}+t^{5}+t^{4}+2 t^{3}=t^{3}(t-1)^{4}\left(t^{2}+2\right)$

9. $\quad$ III II $3^{2}$ does not exist, G

10. III II $321 \quad y^{2}=x^{3}+t \in x^{2}+t\left(2 t^{2}+2\right) x+\frac{t^{2}}{\epsilon}\left((1-i) t^{3}+t^{2}+i t+i\right)$ $-\Delta=t^{9}+(1+i) t^{8}+(2+i) t^{7}+t^{6}+(2-i) t^{5}+(1-i) t^{4}+t^{3}=t^{3}(t-$ $\left.1)^{3}(t-i)^{2}(t+1)\right)$

11. IIIII $31^{3} \quad y^{2}=x^{3}+t x^{2}+t\left(t^{2}+1\right) x+t^{2}\left(t^{3}+t^{2}\right)$

$-\Delta=2 t^{9}+2 t^{7}+2 t^{6}+t^{4}+2 t^{3}=-t^{3}(t-1)^{3}\left(t^{3}+t+2\right)$

12. III II $2^{3} \quad y^{2}=x^{3}+t x^{2}+t\left((1-i) t^{2}+1+i\right) x+t^{2}\left(-i t^{3}+2 t^{2}+(1+i) t+2\right)$

$-\Delta=(-1-i) t^{9}-i t^{8}+t^{7}-i t^{6}+t^{5}+2 i t^{4}+(-1+i) t^{3}=t^{3}(t-1)^{2}(t+$ $1)^{2}\left(-1+i-i t+(-1-i) t^{2}\right)$

13. III II $2^{2} 1^{2} \quad y^{2}=x^{3}+t x^{2}+t\left(t^{2} i+1\right) x+t^{2}\left(t^{3}+(1-i) t^{2}-(1+i) t+1-i\right)$

$-\Delta=t^{9} i+t^{8}+(2+i) t^{7}+t^{6}-(1+2 i) t^{5}+t^{4}-t^{3}=t^{3}(t-1)^{2}(t+1)^{2}\left(2+t+i t^{2}\right)$

14. III II $21^{4} \quad y^{2}=x^{3}+t x^{2}+t\left(t^{2}+1\right) x+t^{2}\left(t^{3}+2+2 t\right)$

$-\Delta=2 t^{9}+t^{5}+t^{4}+2 t^{3}=t^{3}(t-1)^{2}\left(2+2 t+t^{3}+2 t^{4}\right)$

15. IIIII 1 $1^{6} y^{2}=x^{3}+t x^{2}+t\left(2 t^{2}+2\right) x+t^{2}\left(2 t^{3}+t^{2}+2 t+1\right)$

$-\Delta=t^{9}+2 t^{8}+2 t^{7}+2 t^{5}+t^{4}+t^{3}=t^{3}(t-1)(t+1)(t-i)(t+$ i) $\left(t-\frac{1}{1-i}\right)\left(t-\frac{1}{1+i}\right)$

16. IIIIII 6 does not exist, G

17. IIIIII $51 \quad y^{2}=x^{3}+t \in x^{2}+t^{3} x i+t x+\frac{t^{4}}{\epsilon}+\frac{t^{3}}{(2+2 i) \epsilon}+\frac{2 t^{3} i}{2+2 i) \epsilon}+\frac{2 t^{2}}{\epsilon}$

$-\Delta=t^{9} i-(2+2 i) t^{8}+(1+1 i) t^{7}+\left(\frac{1}{1+i}+2+i\right) t^{6}+(2+2 i) t^{5}+(2+$ $2 i) t^{4}+2 t^{3}$, where $\epsilon^{2}+1+i=0$

18. III III 42 does not exist, G]

19. III III $41^{2} \quad y^{2}=x^{3}+t \in x^{2}+t\left(t^{2}+1+i\right) x+\frac{t^{2}}{\epsilon}\left(-t^{2} i-\left(\frac{1}{2}-\frac{1 i}{2}\right) t+1\right)$ $-\Delta=2 t^{9}-(1+i) t^{8}+(1-i) t^{7}-(1+i) t^{6}-(2+2 i) t^{5}+(2+i) t^{4}+(2+i) t^{3}$, where $\epsilon^{2}+1+i=0$

20. III III $3^{2}$ does not exist, $\mathrm{G}$

21. III III $321 \quad y^{2}=x^{3}+t \in x^{2}+t\left((1-i) t^{2}+2\right) x+\frac{t^{2}}{\epsilon}\left(-t^{2}+\left(\frac{1}{5}-\frac{2 i}{5}\right) t+1\right)$

$-\Delta=(2+2 i) t^{9}+(2+2 i) t^{8}+2 i t^{7}+t^{6}+2 i t^{5}-(1-i) t^{4}+t^{3}$ 
22. IIIIII 3 $1^{3} \quad y^{2}=x^{3}+t x^{2}+t\left(2 t^{2}+1\right) x+t^{2}\left(1+t^{2}+t\right)$

$-\Delta=t^{9}+t^{8}+2 t^{7}+2 t^{5}+t^{4}+2 t^{3}=-t^{3}(t-1)^{3}\left(2 t^{3}+2 t^{2}+t+2\right)$

23. III III 2 $2^{3}=x^{3}+t x^{2}+t\left(2 t^{2}+t+2\right) x+t^{2}\left(1+t^{2}\right)$

$-\Delta=t^{9}+t^{8}+2 t^{6}+t^{4}+t^{3}=t^{3}(t-1)^{2}(t-i)^{2}(t+i)^{2}$

24. III III $2^{2} 1^{2} \quad y^{2}=x^{3}+t x^{2}+t\left(t^{2}+2\right) x+t^{2}\left(t^{2}+2 t+2\right)$

$-\Delta=2 t^{9}+t^{8}+2 t^{7}+2 t^{6}+t^{5}+t^{4}+t^{3}=-t^{3}\left(\frac{t-1}{i}\right)^{2}\left(\frac{t+1}{i}\right)^{2}\left(t-\frac{1}{1+i}\right)\left(t-\frac{1}{1-i}\right)$

25. III III $21^{4} \quad y^{2}=x^{3}+t x^{2}+t\left(2 t^{2}+1\right) x+t^{2}\left(t^{2} i+t i+i\right)$

$-\Delta=t^{9}+2 t^{8}+2 t^{7}+t^{6}+2 t^{5}+2 t^{4}+2 t^{3}=t^{3}(t-1)^{2}\left(-1+t^{3}+t^{4}\right)$

26. III III $1^{6} \quad y^{2}=x^{3}+t x^{2}+t\left(t^{2}+t+1\right) x+t^{2}\left(1+t^{2}\right)$

$-\Delta=2 t^{9}+t^{8}+2 t^{7}+t^{5}+t^{4}+2 t^{3}=-t^{3}(t-1)\left(t^{5}+t^{3}+t^{2}+2\right)$

27. III 9 does not exist, $\mathrm{A}$

28. III 81 does not exist, $\mathbb{F}$

29. III 72 does not exist, $\mathbb{F}$

30. III 7 $1^{2} \quad y^{2}=x^{3}+t(t+1) x^{2}+t\left(2 t^{2}+2\right) x+t^{4}$

$-\Delta=2 t^{5}+t^{4}+t^{3}=t^{3}\left(1+t-t^{2}\right)$

31. III 6 $3 \quad y^{2}=x^{3}+t^{2} x^{2}+t\left(t^{2}+1\right) x+t^{2}\left(2+t^{2}+2 t\right)$

$-\Delta=t^{6}+2 t^{3}=t^{3}(t-1)^{3}$

32. III 621 does not exist, $\mathbb{E}$

33. III $61^{3} \quad y^{2}=x^{3}+t(t+1) x^{2}+t\left(t^{2}+1\right) x+t^{2}\left(t^{2}+t\right)$

$-\Delta=2 t^{6}+2 t^{5}+t^{4}+2 t^{3}=t^{3}\left(2+t-t^{2}-t^{3}\right)$

34. III 54 does not exist, $\mathbb{F}$

35. III 531 does not exist, $\mathbb{F}$

36. III $5 \mathbf{2}^{\mathbf{2}}$ does not exist,

37. III $521^{2} \quad y^{2}=x^{3}+t x^{2}+t\left(-i t^{3}+2\right) x+t\left((1+i) t^{5}+2 t^{4}+(1-i) t^{3}+t^{2} i+t\right)$

$-\Delta=2 i t^{12}+2 t^{10}+(-1-i) t^{9}+t^{8}+2 t^{7}+2 i t^{6}+2 t^{5}+t^{4}+t^{3}$

38. III $51^{4} \quad y^{2}=x^{3}+t(t+1) x^{2}+t\left(2 t^{2}+i\right) x+t^{2}\left(t^{2}+1+i\right)$

$-\Delta=(-1-i) t^{7}+(2+i) t^{6}-i t^{5}+2 t^{4}+t^{3} i=-t^{3}(t-1)(i-(1-i) t-$ $\left.t^{2}+(1+i) t^{3}\right)$

39. III $\mathbf{4}^{\mathbf{2}} \mathbf{1}$ does not exist $\mathrm{G}$

40. III 432 does not exist, $\mathbb{F}$

41. III $431^{2} \quad y^{2}=x^{3}+t x^{2}+t\left((-1-i) t^{3}+1+2 i\right) x+t\left(2 t^{3}+(1-i) t^{2}\right)$

$-\Delta=(1-i) * t^{12}+2 i t^{10}+(2+i) t^{6}-2 i t^{4}-(1+i) t^{3}$

42. III $42^{2} 1 \quad y^{2}=x^{3}+t(t+1) x^{2}+t\left((-1-i) t^{2}+2+2 i\right) x+t^{2}\left(-i t^{2}+t+i\right)$

$-\Delta=-i t^{8}+2 t^{6}+2 i t^{4}+(1-i) t^{3}=-t^{3}(t-1)^{2}\left(t+\frac{1}{i}\right)^{2}(i t+(1-i))$ 
43. III $421^{3} \quad y^{2}=x^{3}+t(t+1) x^{2}+t\left(t^{2}+1\right) x+t^{2}\left(2+t^{2}+t\right)$

$-\Delta=t^{8}+2 t^{6}+t^{4}+2 t^{3}=t^{3}(t-1)^{2}\left(2-t-t^{2}+t^{3}\right)$

44. III $41^{5} \quad y^{2}=x^{3}+t(t+1) x^{2}+t\left(t^{2}+1\right) x+t^{2}\left(1+t^{2}+t\right)$

$-\Delta=2 t^{8}+2 t^{6}+t^{5}+t^{4}+2 t^{3}=t^{3}\left(2+t+t^{2}-t^{3}-t^{5}\right)$

45. III $3^{3} \quad y^{2}=x^{3}+t^{2} x^{2}+t\left(t^{2}+2\right) x+t^{2}\left(1+t^{2}\right)$

$-\Delta=2 t^{9}+t^{6}+t^{3}=-t^{3}\left(t-\frac{1}{(1-i)}\right)^{3}\left(t-\frac{1}{(1+i)}\right)^{3}$

46. III $3^{2} \mathbf{2} 1$ does not exist,

47. III $3^{2} \mathbf{1}^{3} \quad y^{2}=x^{3}+\left(t^{2}+t i\right) x^{2}+\left(\phi_{0} t+t^{3} i\right) x+2 t^{4}+t^{3} \phi_{0}$

$-=\left(2 i+2 \phi_{0}\right) t^{9}+\left(-i \phi_{0}+1\right) t^{8}+\left(2 i+2 \phi_{0}\right) t^{7}+\left(2 i+2 \phi_{0}-i \phi_{0}\right) t^{6}+$ $\left(\phi_{0} i+2\right) t^{5}+\left(i+\phi_{0}\right) t^{4}+\left(2 \phi_{0}+2 i+\phi_{0} i\right) t^{3}=-t^{3}(t-1)^{3}\left(\left(\phi_{0}+i\right) t^{3}+\right.$ $\left.\left(-1+i \phi_{0}\right) t^{2}+\left(i+\phi_{0}\right) t+\left(-\phi_{0}-i+i \phi_{0}\right)\right)$, where $\phi_{0}^{2}+\phi_{0}+i=0$

48. III $32^{3} \quad y^{2}=x^{3}+t(t+1) x^{2}+2 t x+t^{2}(2+2 t)$

$-\Delta=t^{9}+t^{8}+2 t^{6}+t^{4}+t^{3}=t^{3}(t-1)^{2}(t-i)^{2}(t+i)^{2}$

49. III $32^{2} 1^{2} \quad y^{2}=x^{3}+t x^{2}+t\left((1+2 i) t^{3}+1+i\right) x+t\left(t^{5} i+t^{4} i+t^{3}+(1-\right.$ i) $\left.\mathrm{t}^{2}-\mathrm{it}\right)$

$-\Delta=\left((-1-i) t^{12}+i t^{10}+2 i t^{9}+2 i t^{8}+(2+i) t^{6}+i t^{5}+2 i t^{4}+(-1+i) t^{3}=\right.$ $\left.t^{3}(t-1)^{3}\left(t^{6}+(1+i) t^{4}-i\right) t^{3}-(1+2 i) t^{2}+(1+i) t+i\right)$

50. III $321^{4} \quad y^{2}=x^{3}+t(t+1) x^{2}+t\left((1+i) t^{2}+1\right) x+t^{2}\left(-i t^{2}+(1+i) t+1+i\right)$ $-\Delta=(1+i) t^{9}-(2+3 i) t^{8}+(1+2 i) t^{7}+(-1+i) t^{6}+(4-i) t^{5}+t^{4}+2 t^{3}=$ $t^{3}(t-1)^{2}\left(2-t-i t^{2}-i t^{3}+(1+i) t^{4}\right)$

51. III $31^{6} \quad y^{2}=x^{3}+t^{2} x^{2}+t x+t^{2}(1+t)$

$-\Delta=t^{9}+t^{8}+-t^{6}+t^{3}=t^{3}\left(t^{6}+t^{5}-t^{3}+1\right)$

52. III $2^{4} 1 \quad y^{2}=x^{3}+t \in x^{2}+t\left(2 t^{3}+2+i\right) x+\frac{t^{2}}{\epsilon}\left((-1+i) t^{4}-(1+i) t^{3}-\right.$ $\left.(1+i) t^{2}+t i+i\right)$

$-\Delta=t^{12}+(2-i) t^{10}+t^{9}+4 i t^{8}+(2+4 i) t^{7}+(2+4 i) t^{6}+(2+4 i) t^{5}-(2+$ $4 i) t^{4}+(4+4 i) t^{3}=t^{3}(t-(-1-i))^{2}(t-1)^{2}(t+1)^{2}(t+i)^{2}(t-(-1+i))$, where $\epsilon^{2}=2-i$

53. III $2^{3} 1^{3} \quad y^{2}=x^{3}+t^{2} x^{2}+t x+t^{2}\left(2+2 t^{2}+2 t\right)$

$-\Delta=t^{10}+t^{9}+t^{8}+t^{6}+2 t^{3}=t^{3}(t-1)^{2}(t+1)^{2}\left(2+t^{2}+t^{3}\right)$

54. III $2^{2} 1^{5} \quad y^{2}=x^{3}+t^{2} x^{2}+t\left(t^{2}+2\right) x+t^{2}\left(2+2 t^{2}+2 t\right)$

$-\Delta=2 t^{10}+2 t^{8}+t^{6}+t^{3}=t^{3}(t-1)^{2}\left(1-t-t^{3}+t^{4}-t^{5}\right)$

55. III $21^{7} \quad y^{2}=x^{3}+\left(t+t^{2}\right) x^{2}+2 t x+t^{4}+2 t^{3}$

$-\Delta=2 t^{10}+t^{9}+2 t^{7}+2 t^{6}+2 t^{5}+t^{4}+t^{3}=t^{3}(t-i)(t+i)\left(1+t+t^{2}+\right.$ $\left.t^{3}+t^{4}-t^{5}\right)$

56. III $1^{9} \quad y^{2}=x^{3}+t(t+1) x^{2}+2 t x+t^{2}\left(t^{3}+2\right)$

$-\Delta=2 t^{11}+t^{6}+t^{4}+t^{3}=t^{3}(t-i)(t+i)\left(1+t-t^{2}+t^{4}-t^{6}\right)$ 


\subsubsection{Case $3 \mathrm{~A}$}

$$
\begin{gathered}
\delta=5, \quad r=2 \quad \text { Type IV } \\
y^{2}=x^{3}+t c_{1} x^{2}+t^{2} c_{2} x+t^{2} c_{4}, \quad t \nmid c_{4}, t \nmid c_{1} \\
-\Delta=t^{2} c_{1}^{2}\left(t^{3} c_{1} c_{4}-t^{4} c_{2}^{2}\right)+t^{6} c_{2}^{3}
\end{gathered}
$$

1. IV 7 does not exist, $\mathrm{C}$

2. IV 61 does not exist, $\mathbf{F}$

3. IV 52 does not exist, G

4. IV $51^{2} \quad y^{2}=x^{3}+t(t+1) x^{2}+2 t^{2} x+t^{2}$

$-\Delta=2 t^{7}+2 t^{5}=t^{5}\left(2 t^{2}+2\right)^{2}$

5. IV 43 does not exist, $\mathbf{F}$

6. IV $421 \quad y^{2}=x^{3}+t^{2} i+t^{2}\left(x^{2}+x+2 x i+2\right)$

$-\Delta=(1+i) t^{8}+t^{7}+t^{6}-i t^{5}$

7. IV $41^{3} \quad y^{2}=x^{3}+t(t+1) x^{2}+2 t^{2} x i-t^{2}(2+i)$

$-\Delta=-(2+2 i) t^{8}+t^{7}-(1+2 i) t^{6}-(1+2 i) t^{5}$

8. $\quad$ IV $3^{2} 1 \quad y^{2}=x^{3}+t x^{2} i+t^{2}\left(x^{2}+2 i\right)-t^{3} i$

$-\Delta=t^{9} i+t^{8} i+t^{6}+t^{5}$

9. $\quad$ IV $3 \mathbf{2}^{2} \quad y^{2}=-\frac{1}{2} t^{3} i+(2+2 i)\left(-2 x i+x-x^{2} i+2+2 i+x^{2}\right) t^{2}+(2+$ $2 i)\left(x^{2} i+x^{2}\right) t+(2+2 i)\left(x^{3}-x^{3} i\right)$

$-\Delta=-i t^{9}-(1-i) t^{8}+t^{7} i-i t^{6}+t^{5}$

10. IV $321^{2} \quad y^{2}=x^{3}+t x^{2}+t^{2}\left(x^{2}+2 x+1\right)+2 t^{3}$

$-\Delta=t^{9}+2 t^{7}+t^{6}+2 t^{5}$

11. IV $31^{4} \quad y^{2}=x^{3}+t(t+1) x^{2}+2 t^{2} x+t^{2}(t+1)$

$-\Delta=2 t^{9}+2 t^{7}+2 t^{6}+2 t^{5}$

12. $\quad$ IV $2^{3} 1 \quad y^{2}=x^{3}+t(t+1) x^{2}+2 t^{2} x+t^{2}\left((2+i) t^{2}+(2+2 i) t+2+2 i\right)$

$-\Delta=(1+2 i) t^{10}+(1+i) t^{9}+(2+i) t^{8}+(2+i) t^{7}+(2-i) t^{6}+(1-i) t^{5}$

13. IV 2 $2^{2} 1^{3} \quad y^{2}=x^{3}+t(t+1) x^{2}+2 t^{2} x+t^{2}\left(t^{3} \gamma_{3}+2 t^{2}+t\right)$

$-\Delta=t^{10}+2 t^{9}+t^{8}+2 t^{6}$

14. IV $21^{5} \quad y^{2}=x^{3}+t(t+1) x^{2}+t^{2} x+t^{2}\left(t^{2}+2 t+1\right)$

$-\Delta=2 t^{10}+t^{9}+t^{7}+2 t^{5}$

15. IV $1^{7} \quad y^{2}=x^{3}+t(t+1) x^{2}+t^{2} x+t^{2}\left(2 t^{3}+t^{2}+1\right)$

$-\Delta=t^{11}+2 t^{10}+t^{8}+t^{7}+2 t^{6}+2 t^{5}$

16. IV IV $2 y^{2}=x^{3}+t x^{2}+2 t^{2} x+t^{2}\left(2 t^{2}+2 t+2\right)$

$-\Delta=t^{7}+t^{6}+t^{5}=t^{5}(t-1)^{2}$ 
17. IV IV $1^{2} \quad y^{2}=x^{3}+t x^{2}+2 t^{2} x+t^{2}\left(2+t^{2}\right)$

$-\Delta=2 t^{7}+t^{5}=2 t^{5}(t-1)(t+1)$

18. IV II 3 $y^{2}=x^{3}+t x^{2}+2 t^{2} x+t^{2}\left(t^{3}+2\right)$

$-\Delta=2 t^{8}+t^{5}=t^{5}(t-1)^{2}$

19. IV II 21 $\mathrm{y}^{2}=\mathrm{x}^{3}+\mathrm{t} \mathrm{x}^{2}+2 \mathrm{t}^{3} \phi_{1} \mathrm{x}+\mathrm{t}^{2}\left(2 \mathrm{t}^{3}+\mathrm{t}^{2}+\mathrm{t}+2\right)$

$-\Delta=t^{8}+2 t^{7}+2 t^{6}+t^{5}$

20. IV II $1^{3} \quad y^{2}=x^{3}+t x^{2}+t^{2}\left(2 t^{3}+t+2\right)$

$-\Delta=t^{8}+2 t^{6}+t^{5}$

21. IV III $4 y^{2}=x^{3}+t x^{2} i+t^{3} x+t^{2}(t i+2 i)$

$-\Delta=t^{9}+2 t^{8}+2 t^{6}+t^{5}=t^{5}(t-1)^{4}$

22. IV III $31 y^{2}=x^{3}+t x^{2} i+2 t^{3} x+t^{2}(2 t i+2 i)$

$-\Delta=2 t^{9}+2 t^{8}+t^{6}+t^{5}=-t^{5}(t-1)^{3}(t+1)$

23. IV III $\left.2^{2} \quad y^{2}=x^{3}+2 t x^{2}+2 t^{2}(1+i) t+1\right) x+t^{2}\left((2+2 i) t^{2}+2 t+1+2 i\right)$

$-\Delta=-(1-i) t^{9}+2 i t^{8}-(2+2 i) t^{7}+2 t^{6}-(2+i) t^{5}$

24. IV III $21^{2} \quad y^{2}=x^{3}+t x^{2}+2 t^{2}(2 t+1) x+t^{2}\left(2 t^{2}+2 t+2\right)$

$-\Delta=t^{9}+t^{8}+2 t^{7}+t^{6}+t^{5}=t^{5}(t-1)^{2}(t-i)(t+i)$

25. IV III $1^{4} \quad y^{2}=x^{3}+t x^{2}+2 t^{2}(t+2) x+t^{2}\left(t^{2}+t+2\right)$

$-\Delta=2 t^{9}+t^{8}+t^{6}+t^{5}=t^{5}\left(2 t^{4}+t^{3}+t+1\right)$

26. IV II $4 \quad \mathrm{y}^{2}=\mathrm{x}^{3}+\mathrm{t} \mathrm{x}^{2}+2 \mathrm{t}^{2}(2 \mathrm{t}+1) \mathrm{x}+\mathrm{t}^{2}\left(2 \mathrm{t}^{3}+\mathrm{t}^{2}+\mathrm{t}+2\right)$

$-\Delta=t^{9}+2 t^{8}+2 t^{6}+t^{5}=t^{5}(t-1)^{4}$

27. IV II $31 \quad y^{2}=x^{3}+t x^{2}+2 t^{2}(t+1) x+t^{2}\left(2 t^{3}+2 t^{2}+2 t+2\right)$

$-\Delta=2 t^{9}+2 t^{8}+t^{6}+t^{5}=t^{5}(t-1)^{3}(t+1)$

28. IV II $2^{2} \quad y^{2}=x^{3}+t x^{2}+2 t^{2}(2 t+1) x+t^{2}\left(t^{3}+2\right)$

$-\Delta=t^{9}+t^{7}+t^{5}=t^{5}(t-1)^{2}(t+1)^{2}$

29. IV II $2 \mathbf{1}^{2} \quad y^{2}=x^{3}+t x^{2} i+t^{3} x+t^{2}\left(t^{3} i+t^{2} i+2 t i+2 i\right)$

$-\Delta=t^{9}+t^{8}+2 t^{7}+t^{6}+t^{5}=t^{5}(t-1)^{2}(t-i)(t+i)$

30. IV II 1 $1^{4} y^{2}=x^{3}+t x^{2}+2 t^{2}(t+1) x+t^{2}\left(2 t^{3}+2 t^{2}+t+2\right)$

$-\Delta=2 t^{9}+2 t^{8}+2 t^{6}+t^{5}=t^{5}\left(2 t^{4}+2 t^{3}+2 t+1\right)$

\subsubsection{Case 3B}

$$
\begin{gathered}
\delta=6, \quad r=2 \quad \text { Type IV } \\
y^{2}=x^{3}+t^{2} c_{0} x^{2}+t^{2} c_{2} x+t^{2} c_{4}, \quad t \nmid c_{4}, t \nmid c_{2} \\
-\Delta=t^{4} c_{0}^{2}\left(t^{4} c_{0} c_{4}-t^{4} c_{2}^{2}\right)+t^{6} c_{2}^{3}
\end{gathered}
$$


1. IV $6 \quad y^{2}=x^{3}+t^{2} x^{2}+\left(t^{4}+t^{3}+2 t^{2}\right) x+2 t^{6}+2 t^{5}+2 t^{3}+t^{2}$ $-\Delta=2 t^{12}+2 t^{9}+2 t^{6}=2 t^{6}\left(t^{6}+t^{3}+1\right)$

2. IV 51 does not exist, $\mathrm{G}$

3. IV 42 does not exist, G

4. IV $41^{2} \quad y^{2}=x^{3}+t^{2} x^{2}+\left(t^{4}+t^{3}+t^{2}\right) x+2 t^{6}+t^{5}+t^{3}$

$-\Delta=2 t^{12}+2 t^{11}+2 t^{8}+t^{6}=t^{6}\left((t-1)^{4}(t-(-1-i))(t-(-1+i))\right.$

5. $\quad$ IV $3^{2}$ does not exist, $G$

6. IV 321 does not exist, G

7. IV $31^{3} \quad y^{2}=x^{3}+2 t^{2} x^{2}+\left(t^{3}+t^{2}\right) x+2 t^{6}+t^{5}+2 t^{4}+t^{3}+t^{2}$

$-\Delta=2 t^{12}+2 t^{11}+t^{9}+t^{8}+t^{6}=-t^{6}\left(t^{3}+2\right)\left(2 t^{3}+t^{2}+1\right)$

8. $\quad \mathbf{I V ~ 2}^{\mathbf{3}}$ does not exist, $\mathrm{G}$

9. IV $2^{2} 1^{2} \quad y^{2}=x^{3}+t^{2} x^{2}+\left(t^{3}+2 t^{2}\right) x+2 t^{6}+2 t^{4}+2 t^{2}$

$-\Delta=2 t^{12}+t^{10}+t^{8}+2 t^{6}=t^{6}\left(1+t^{2}\right)\left(2 t^{4}+2 t^{2}+2\right)$

10. IV $21^{4} \quad y^{2}=x^{3}+t^{2} x^{2}+t^{2}\left(t^{2}+t+1\right) x+t^{2}\left(2 t^{4}+t^{2}+2 t+1\right)$

$-\Delta=2 t^{6}+t^{5}+t^{4}+t^{3}+1=-t^{6}(t-1)^{2}(t-i)(t-(1-i))(t-(1+i))$

11. IV $1^{6} \quad y^{2}=x^{3}+t^{2} x^{2} i+t^{2}\left(t^{2}+t+1\right) x+t^{2}\left(3 i t^{4}-2 i t^{3}-2 i t^{2}+i\right)$

$-\Delta=2 t^{12}+t^{10}+2 t^{8}+t^{6}=-t^{6}(t-1)(t+1)\left(t^{4}+1\right)$

12. IV II $y^{2}=x^{3}+t^{2} x+t^{2}\left(t^{3}+1+t\right)$

$-\Delta=2 t^{6}$

13. IV IIII $\mathrm{y}^{2}=\mathrm{x}^{3}+\mathrm{t}^{2}(2 \mathrm{t}+1) \mathrm{x}+\mathrm{t}^{2}\left(\mathrm{t}^{3}+\mathrm{t}+1\right)$

$-\Delta=t^{9}-t^{6}=2 t^{6}(2 t+1)^{3}$

14. IV III II $y^{2}=x^{3}+t^{2}(2 t+1) x+t^{2}(2 t+1)$

$-\Delta=t^{9}-t^{6}=2 t^{6}(2 t+1)^{3}$

15. IV III III $y^{2}=x^{3}+t^{2}(2 t+1) x$

$-\Delta=t^{9}-t^{6}=2 t^{6}(2 t+1)^{3}$

16. IV IV $y^{2}=x^{3}+2 t^{2} x+t^{2}\left(t^{2}+t+1\right)$

$-\Delta=\mathrm{t}^{6}$

4.2.10 Case 3C

$$
\begin{gathered}
\delta=8, \quad r=2 \quad \text { Type IV } \\
y^{2}=x^{3}+t^{2} c_{0} x^{2}+t^{3} c_{1} x+t^{2} c_{4}, \quad t \nmid c_{4}, t \nmid c_{0} \\
-\Delta=t^{4} c_{0}^{2}\left(t^{4} c_{0} c_{4}-t^{6} c_{1}^{2}\right)+t^{9} c_{1}^{3}
\end{gathered}
$$

1. IV $4 \quad y^{2}=x^{3}+2 t^{2} x^{2}+t^{2}\left(t^{4}+2 t^{3}+2 t+1\right)$

$\Delta=t^{7}\left(t^{5}+2 t^{4}+2 t^{2}+t\right)=t^{12}+2 t^{11}+2 t^{9}+t^{8}$ 
2. IV $31 \quad y^{2}=x^{3}+t^{2} x^{2}+t^{3} x+t^{2}\left(2 t^{4}+2 t^{3}+t^{2}+1\right)$

$$
\Delta=t^{12}+t^{11}+2 t^{8}+2 t^{9}
$$

3. IV 2 $\mathbf{2}^{2} \quad y^{2}=x^{3}+t^{2} x^{2}+t^{3}(t+2) x+t^{2}\left(2 t^{4}+t^{3}+2+1\right)$ $\Delta=\mathrm{t}^{12}+\mathrm{t}^{8}+\mathrm{t}^{10}$

4. $\quad$ IV 2 $1^{2} \quad y^{2}=x^{3}+t^{2} x^{2}+t^{3}(t+1) x+t^{2}\left(2 t^{4}+t^{3}+2 t^{2}+t+2\right)$ $\Delta=2 t^{7}\left(2 t^{5}+t\right)=t^{12}-t^{8}$

5. IV $1^{4} \quad y^{2}=x^{3}+t^{2} x^{2}+t^{2}\left(2 t^{4}+1\right)$ $-\Delta=t^{8}\left(2 t^{4}+2 t^{3}+t^{2}+2 t+2\right)$

6. IV II does not exist, $\mathbb{E}$

7. IV II 1 does not exist, $\mathbb{E}$

8. IV III 1 does not exist, $\mathbb{E}$

\subsubsection{Case 3D}

$$
\begin{gathered}
\delta=9, \quad r=2 \quad \text { Type IV } \\
y^{2}=x^{3}+t^{3} c_{1} x+t^{2} c_{4}, \quad t \nmid c_{4}, t \nmid c_{1} \\
-\Delta=t^{9} c_{1}^{3}
\end{gathered}
$$

1. IV 3 does not exist, $\mathbb{E}$

2. IV 21 does not exist, $\mathrm{E}$

3. $\quad \mathbf{I V}^{\mathbf{3}}$ does not exist, $\mathrm{E}$

4. IV II $y^{2}=x^{3}+t^{3}(2 t+1) x+t^{2}$ $-\Delta=t^{9}(2 t+1)^{3}$

5. IV III $y^{2}=x^{3}+t^{3}(2 t+1) x+t^{2}\left(t^{2}+t+1\right)$ $-\Delta=\mathrm{t}^{9}(2 \mathrm{t}+1)^{3}$

\subsubsection{Case 3E}

$$
\begin{gathered}
\delta=12, \quad r=2 \quad \text { Type } \mathbf{I V} \\
\mathrm{y}^{2}=\mathrm{x}^{3}+\mathrm{t}^{4} \mathrm{c}_{0} \mathrm{x}+\mathrm{t}^{2} \mathrm{c}_{4}, \quad \mathrm{t} \nmid \mathrm{c}_{4}, \mathrm{t} \nmid \mathrm{c}_{0} \\
-\Delta=\mathrm{t}^{12} \mathrm{c}_{0}^{3}
\end{gathered}
$$




\subsubsection{Case 4}

In 3 there are two different sub-cases to this case, but both have Weierstrass

form and discriminant as listed below, and thus we may treat them together.

$$
\begin{gathered}
\delta=6, \quad r=4 \quad \text { Type } \mathbf{I}_{0}^{*} \\
y^{2}=x^{3}+t c_{1} x^{2}+t^{2} c_{2} x+t^{3} c_{3} \\
-\Delta=t^{2} c_{1}^{2}\left(t^{4} c_{1} c_{3}-t^{8} c_{0}^{2}\right)+t^{12} c_{0}^{2}
\end{gathered}
$$

1. $\mathbf{I}_{\mathbf{0}}^{*} \mathbf{6}$ does not exist, $\mathrm{Q}$

2. $\quad \mathbf{I}_{\mathbf{0}}^{*} \mathbf{5} \mathbf{1}$ does not exist, $\mathrm{C}$

3. $\quad \mathbf{I}_{\mathbf{0}}^{*} \mathbf{4 2}$ does not exist, $\mathrm{C}$

4. $\quad I_{0}^{*} 41^{2} \quad y^{2}=x^{3}+t^{2} x^{2}+2 t^{2} x+t^{3}\left(t^{3}+t^{2}\right)$

$-\Delta=t^{4}\left(t^{5}\left(t^{3}+t^{2}\right)+2 t^{4}\right)+2 t^{6}=t^{6}\left(t^{6}+t^{5}+2 t^{2}+2\right)$

5. $\quad \mathbf{I}_{\mathbf{0}}^{*} \mathbf{3}^{\mathbf{2}}$ does not exist, $\mathrm{C}$

6. $\quad \mathbf{I}_{\mathbf{0}}^{*} \mathbf{3 2 1}$ does not exist, $\mathrm{C}$

7. $\mathbf{I}_{0}^{*} 3 \mathbf{1}^{3} \quad \mathrm{y}^{2}=\mathrm{x}^{3}+\mathrm{t} \mathrm{x}^{2}+\mathrm{t}^{4} x+\mathrm{t}^{3}\left(\mathrm{t}^{3}+\mathrm{t}+1\right)$

$-\Delta=t^{2}\left(t^{4}\left(t^{3}+t+1\right)+2 t^{8}\right)+t^{12}=t^{6}\left(t^{3}+2 t+2\right)\left(t^{3}+2\right)$

$\begin{array}{ll}\text { 8. } & \mathbf{I}_{0}^{*} 2^{3} \quad y^{2}=\left(\frac{1}{\epsilon}+\frac{i}{\epsilon}\right) t^{6}-\frac{i t^{5}}{\epsilon}\left(2 x+\frac{1-i}{\epsilon}\right) t^{4}-\frac{t^{3}}{\epsilon}+t x^{2} \epsilon+x^{3} \\ -\Delta=t^{12}+i t^{10^{6}}+(1-i) t^{9}+2 t^{8}-(1+i) t^{7}-2 i t^{6}=t^{6}(t+1+i)^{2}(t-1)^{2}(t-i)^{2}\end{array}$

9. $\quad \mathbf{I}_{0}^{*} \mathbf{2}^{2} \mathbf{1}^{2} \quad \mathrm{y}^{2}=\mathrm{x}^{3}+\mathrm{t}^{2} \chi^{2} i+t^{2}\left(\mathrm{t}^{2}+\mathrm{t}+2\right) x+\mathrm{t}^{3}\left(\mathrm{t}^{2} i-t i+\mathfrak{i}\right)$

$-\Delta=2 t^{12}+t^{10}+t^{8}+2 t^{6}=t^{6}\left(2 t^{6}+t^{4}+t^{2}+2\right)$

10. $\mathbf{I}_{0}^{*} 21^{4} \quad y^{2}=x^{3}+t x^{2}+t^{2}\left(t^{2}+t+1\right) x+t^{3}\left(2 t^{2}+2 t+2\right)$

$-\Delta=-t^{12}+t^{10}+t^{9}+t^{8}+t^{6}=t^{6}(t-1)^{2}\left(-t^{4}+t^{3}+t^{2}-t+1\right)$

11. $\mathbf{I}_{0}^{*} \mathbf{1}^{6} \quad \mathrm{y}^{2}=\mathrm{x}^{3}+\mathrm{t} \mathrm{x}^{2}+\mathrm{t}^{2}\left(2 \mathrm{t}^{2}+2 \mathrm{t}+1\right) \mathrm{x}+\mathrm{t}^{3}\left(\mathrm{t}^{2}+\mathrm{t}+2\right)$

$-\Delta=t^{12}+t^{10}+t^{8}+t^{6}=t^{6}(t-i)(t+i)\left(t^{4}-1\right)$

12. $\quad \mathbf{I}_{\mathbf{0}}^{*} \mathbf{I}_{\mathbf{0}}^{*} \quad y^{2}=x^{3}+2 t 2 x+t^{3}$

$-\Delta=t^{6}$

13. $\mathbf{I}_{0}^{*} \mathbf{I V} \mathrm{t}^{6} \mathrm{y}^{2}=\mathrm{x}^{3}+2 \mathrm{t}^{2} \mathrm{x}+\mathrm{t}^{3}(\mathrm{t}+1)$

14. $\mathbf{I}_{\mathbf{0}}^{*}$ II $\mathrm{y}^{2}=\mathrm{x}^{3}+2 \mathrm{t}^{2} \mathrm{x}+\mathrm{t}^{3}\left(\mathrm{t}^{2}+\mathrm{t}+1\right)$

$-\Delta=\mathrm{t}^{6}$

15. I I II II $y^{2}=x^{3}+t^{2}(2 t+1) x+t^{3}\left(t^{2}+2 t\right)$

$-\Delta=t^{9}+2 t^{6}=t^{6}\left(t^{3}+2\right)$

16. I I III III $y^{2}=x^{3}+t^{2}(2 t+1) x$

$-\Delta=t^{9}+2 t^{6}=t^{6}\left(t^{3}+2\right)$ 
17. $\mathbf{I}_{0}^{*}$ II III $\mathrm{y}^{2}=\mathrm{x}^{3}+\mathrm{t}^{2}(2 \mathrm{t}+1) \mathrm{x}+\mathrm{t}^{3}(\mathrm{t}+2)$

$-\Delta=t^{9}+2 t^{6}=t^{6}\left(t^{3}+2\right)$

18. $\quad \mathbf{I}_{0}^{*} \mathbf{I V} \mathbf{1} \mathrm{y}^{2}=\mathrm{x}^{3}+\mathrm{t} \mathrm{x}^{2}+\mathrm{t}^{2} \mathrm{x}+\mathrm{t}^{3}(\mathrm{t}+2)$

$-\Delta=-t^{7}+t^{6}=-t^{6}(t-1)$

19. $\mathbf{I}_{0}^{*} \mathbf{I I} 2 \mathrm{y}^{2}=\mathrm{x}^{3}+\mathrm{t} \mathrm{x}^{2}+\mathrm{t}^{3}\left(\mathrm{t}^{2}+\mathrm{t}+1\right)$

$-\Delta=\mathrm{t}^{8}+\mathrm{t}^{7}+\mathrm{t}^{6}=\mathrm{t}^{6}(\mathrm{t}-1)^{2}$

20. $\quad \mathbf{I}_{0}^{*} \mathbf{I I} \mathbf{1}^{2} \quad \mathrm{y}^{2}=\mathrm{x}^{3}+\mathrm{t} \mathrm{x}^{2}+\mathrm{t}^{3}\left(\mathrm{t}^{2}-1\right)$

$-\Delta=t^{8}-t^{6}=t^{6}(t-1)(t+1)$

21. $\mathbf{I}_{\mathbf{0}}^{*}$ III $3 \mathrm{y}^{2}=\mathrm{x}^{3}+\mathrm{t} \mathrm{x}^{2}+\mathrm{t}^{6}+\mathrm{t}^{3}$

$-\Delta=t^{9}+t^{6}=t^{6}(t+1)^{3}$

22. $\quad \mathbf{I}_{0}^{*}$ III $21 \quad \mathrm{y}^{2}=\mathrm{x}^{3}+\mathrm{t}^{2} \mathrm{i}+\mathrm{t}^{2}(2 \mathrm{t}+1) \mathrm{x}$

$-\Delta=t^{9}+2 t^{8}+2 t^{7}+t^{6}=t^{6}(t-1)^{2}(t+1)$

23. $\mathbf{I}_{0}^{*}$ III 1 $^{3} \quad \mathrm{y}^{2}=\mathrm{x}^{3}+\mathrm{t} \mathrm{x}^{2}+\mathrm{t}^{2}(\mathrm{t}+1) \mathrm{x}+2 \mathrm{t}^{3}$

$-\Delta=2 t^{9}+t^{8}+2 t^{7}+t^{6}=-t^{6}(t-1)\left(t-\frac{1}{i}\right)\left(t+\frac{1}{i}\right)$

24. I I II $3 \quad y^{2}=x^{3}+t x^{2}+t^{2}(t+1) x+t^{3}\left(t^{2}+2 t+2\right)$

$-\Delta=2 t^{9}+t^{6}=-t^{6}(t-1)^{3}$

25. $\quad \mathbf{I}_{0}^{*} \mathbf{I I} 2 \mathbf{1} \mathrm{y}^{2}=\mathrm{x}^{3}+\mathrm{tx}^{2}+\mathrm{t}^{2}(2 \mathrm{t}+1) \mathrm{x}+\mathrm{t}^{3}\left(2 \mathrm{t}^{2}+2 \mathrm{t}+2\right)$

$-\Delta=t^{9}+2 t^{8}+2 t^{7}+t^{6}=t^{6}(t-1)^{2}(t+1)$

26. $\mathbf{I}_{0}^{*} \mathbf{I I} \mathbf{1}^{3} \quad \mathrm{y}^{2}=\mathrm{x}^{3}+\mathrm{tx}^{2} \mathrm{i}+\mathrm{t}^{2}(\mathrm{t}+1) \mathrm{x}+\mathrm{t}^{3}\left(\mathrm{t}^{2} \mathrm{i}-\mathrm{ti}\right)$

$-\Delta=2 t^{9}+t^{8}+2 t^{7}+t^{6}=-t^{6}(t-1)(t-i)(t+i)$

\subsubsection{Case 5A}

$$
\begin{gathered}
\delta=7, \quad r=5 \quad \text { Type } \mathbf{I}_{1}^{*} \\
y^{2}=x^{3}+t c_{1} x^{2}+t^{4} c_{0} x+t^{4} c_{2}, \quad t \nmid c_{1}, t \nmid c_{2} \\
-\Delta=t^{2} c_{1}^{2}\left(t^{5} c_{1} c_{2}-t^{8} c_{0}^{2}\right)+t^{12} c_{0}^{3}
\end{gathered}
$$

1. $\mathbf{I}_{\mathbf{1}}^{*} \mathbf{5}$ does not exist, $\mathrm{A}$

2. $\mathbf{I}_{1}^{*} \mathbf{4 1}$ does not exist, $\mathrm{G}$

3. $\quad \mathbf{I}_{\mathbf{1}}^{*} \mathbf{3 2}$ does not exist, $\mathrm{C}$

4. $\quad \mathbf{I}_{1}^{*} 3 \mathbf{1}^{2} \quad \mathrm{y}^{2}=\mathrm{x}^{3}+\mathrm{t} \mathrm{x}^{2}+2 \mathrm{t}^{4} \mathrm{x}+\mathrm{t}^{4}\left(\mathrm{t}^{2}+1\right)$

$\left.-\Delta=t^{2}\left(t^{5}\left(t^{2}+1\right)+2 t^{8}\right)+2 t^{12}\right)=t^{7}\left(t^{2}+1\right)\left(2 t^{3}+1\right)$

5. $\quad \mathbf{I}_{1}^{*} \mathbf{2 1}^{3} \quad \mathrm{y}^{2}=\mathrm{x}^{3}+\mathrm{t}(\mathrm{t}+1) \mathrm{x}^{2}+i \mathrm{t}^{4} x+\mathrm{t}^{4}((1+i) t+i)$

$\left.-\Delta=t^{2}(t+1)^{2}\left(t^{5}(t+1)((1+i) t+i)+t^{8}\right)-t^{12} i\right)=t^{7}(2+i)\left(2 t^{3}+i t^{2}+\right.$ $i t+t+2+i)\left(t^{2}+t+1\right)$

6. $\quad \mathbf{I}_{1}^{*} \mathbf{2}^{2} \mathbf{1} \quad \mathrm{y}^{2}=\mathrm{x}^{3}+\mathrm{t}(\mathrm{t}+1) \mathrm{x}^{2}+\mathrm{t}^{4} \alpha x+\mathrm{t}^{4}\left(\left(2 \alpha^{3}+2 \alpha^{2}\right) \mathrm{t}+2 \alpha^{3}\right)$ $-\Delta=\mathrm{t}^{2}(\mathrm{t}+1)^{2}\left(\mathrm{t}^{5}(\mathrm{t}+1)\left(\left(2 \alpha^{3}+2 \alpha^{2}\right)+2 \alpha^{3}\right)+2 \mathrm{t}^{8} \alpha^{2}\right)+\mathrm{t}^{12} \alpha^{3}=\mathrm{t}^{7} \alpha^{2}\left(2 \alpha \mathrm{t}^{4}+\right.$ $\left.2 \alpha t^{3}+2 t^{3}+2 t^{5}+2 \alpha t+2 t+2 \alpha+\alpha t^{5}\right)$, where $\alpha^{4}+\alpha+1=0$ 
7. $\quad \mathbf{I}_{1}^{*} \mathbf{1}^{5} \quad \mathrm{y}^{2}=\mathrm{x}^{3}+\mathrm{t}(\mathrm{t}+1) \mathrm{x}^{2}+\mathrm{t}^{4} x+\mathrm{t}^{4}\left(\mathrm{t}^{2}+2 t+1\right)$

$\left.-\Delta=t^{2}(t+1)^{2}\left(t^{5}(t+1)\left(t^{2}+2 t+1\right)+2 t^{8}\right)+t^{12}\right)=t^{7}\left(t^{5}+t^{2}+2 t+1\right)$

8. $\quad \mathbf{I}_{1}^{*} \mathbf{I I} \mathbf{1}^{2} \quad \mathrm{y}^{2}=\mathrm{x}^{3}+\mathrm{t}(\mathrm{t}+2) \mathrm{x}^{2}+\mathrm{t}^{4}\left(\mathrm{t}^{2}+1\right)$

$-\Delta=t^{7}(t+2)^{3}\left(t^{2}+1\right)$

9. $\quad \mathbf{I}_{1}^{*}$ II $2 \quad \mathrm{y}^{2}=\mathrm{x}^{3}+\mathrm{t}(\mathrm{t}+2) \mathrm{x}^{2}+\mathrm{t}^{4}\left(2 \mathrm{t}^{2}+\mathrm{t}+2\right)$

$-\Delta=t^{7}(t+2)^{3}\left(2 t^{2}+t+2\right)$

10. $\mathbf{I}_{1}^{*} \mathbf{I I} \mathbf{1} \quad \mathrm{y}^{2}=\mathrm{x}^{3}+\mathrm{t}(\mathrm{t}+2) \mathrm{x}^{2}+\mathrm{t}^{4}\left(2 \mathrm{t}^{2}+1\right)$

$-\Delta=t^{7}(t+2)^{3}\left(2 t^{2}+1\right)$

11. $\mathbf{I}_{1}^{*} \mathbf{I V} \mathrm{y}^{2}=\mathrm{x}^{3}+\mathrm{t}(\mathrm{t}+2) \mathrm{x}^{2}+\mathrm{t}^{4}\left(\mathrm{t}^{2}+\mathrm{t}+1\right)$

$-\Delta=t^{7}(t+2)^{3}\left(t^{2}+t+1\right)$

12. I III 2 does not exist, G

\subsubsection{Case 5B}

$$
\begin{gathered}
\delta=8, \quad r=6 \quad \text { Type } \mathbf{I}_{2}^{*} \\
y^{2}=x^{3}+t c_{1} x^{2}+t^{4} c_{0} x+t^{5} d_{1}, \quad t \nmid c_{1}, t \nmid d_{1} \\
-\Delta=t^{2} c_{1}^{2}\left(t^{6} c_{1} d_{1}-t^{8} c_{0}^{2}\right)+t^{12} c_{0}^{3}
\end{gathered}
$$

1. $\mathbf{I}_{2}^{*} \mathbf{4}$ does not exist, A

2. $\mathbf{I}_{\mathbf{2}}^{*} \mathbf{3} \mathbf{1}$ does not exist, $\mathrm{C}$

3. $\quad \mathrm{I}_{2}^{*} 2^{2} \quad \mathrm{y}^{2}=x^{3}+t x^{2}+2 t^{4} x+2 t^{5}$

$-\Delta=t^{12}+t^{10}+t^{8}=t^{8}(t-1)^{2}(t+1)^{2}$

4. $\quad I_{2}^{*} 21^{2} \quad y^{2}=x^{3}+t x^{2}+t^{4} x+t^{5}(t+2)$

$-\Delta=2 t^{12}+t^{10}+2 t^{9}+t^{8}=t^{8}(t-1)^{2}\left(2 t^{2}+t+1\right)$

5. $\quad \mathbf{I}_{2}^{*} \mathbf{1}^{4} \quad \mathrm{y}^{2}=\mathrm{x}^{3}+\mathrm{t} \mathrm{x}^{2}+\mathrm{t}^{4} \mathrm{x}+\mathrm{t}^{5}(2 \mathrm{t}+1)$

$-\Delta=2 t^{12}+t^{10}+t^{9}+2 t^{8}=t^{8}(t-1)\left(2 t^{3}+t^{2}+1\right)$

6. $\quad \mathbf{I}_{2}^{*} \mathbf{I I} \quad y^{2}=x^{3}+t(t+2) x^{2}+t^{5}(2 t+1)$

$-\Delta=t^{8}(t+2)^{3}(2 t+1)$

7. $\mathbf{I}_{2}^{*} \mathbf{I I} \mathbf{1} \mathrm{y}^{2}=\mathrm{x}^{3}+\mathrm{t}(\mathrm{t}+2) \mathrm{x}^{2}+\mathrm{t}^{5}(2 \mathrm{t}+2)$

$-\Delta=t^{8}(t+2)^{3}(2 t+2)$

\subsubsection{Case 5C}

$$
\begin{gathered}
\delta=9, \quad r=7 \quad \text { Type } \mathbf{I}_{3}^{*} \\
y^{2}=x^{3}+t c_{1} x^{2}+t^{4} c_{0} x+t^{6} d_{0}, t \nmid c_{1}, t \nmid d_{0} \\
-\Delta=t^{2} c_{1}^{2}\left(t^{7} c_{1} d_{0}-t^{8} c_{0}^{2}\right)+t^{12} c_{0}^{3}
\end{gathered}
$$


1. $\mathbf{I}_{\mathbf{3}}^{*} \mathbf{3}$ does not exist, $\mathrm{A}$

2. $\quad \mathbf{I}_{\mathbf{3}}^{*} \mathbf{1 2}$ does not exist, $\mathrm{C}$

3. $\mathbf{I}_{3}^{*} \mathbf{1}^{3} \quad \mathrm{y}^{2}=x^{3}+\mathrm{t} \mathrm{x}^{2}+2 \mathrm{t}^{4} x+2 \mathrm{t}^{6}$

$-\Delta=t^{12}+t^{10}+t^{9}=t^{9}(t-1)\left(t^{2}+t+2\right)$

4. $\quad \mathbf{I}_{3}^{*} \mathbf{I I} \quad \mathrm{y}^{2}=\mathrm{x}^{3}+\mathrm{t}(\mathrm{t}+2) \mathrm{x}^{2}+2 \mathrm{t}^{6}$

$-\Delta=2 t^{9}(t+2)^{3}$

5. $\quad \mathbf{I}_{\mathbf{3}}^{*}$ III does not exist, $\mathbf{C}$

4.2.17 Case 5D

$$
\begin{gathered}
\delta=10, \quad r=8 \quad \text { Type } \mathbf{I}_{4}^{*} \\
y^{2}=x^{3}+t c_{1} x^{2}+t^{4} c_{0} x \quad t \nmid c_{0}, t \nmid c_{1} \\
-\Delta=-t^{10} c_{1}^{2} c_{0}^{2}+t^{12} c_{0}^{3}
\end{gathered}
$$

1. $\quad \mathbf{I}_{\mathbf{4}}^{*} \mathbf{2}$ does not exist, $\mathrm{A}$

2. $\mathbf{I}_{4}^{*} \mathbf{1}^{2} \quad \mathrm{y}^{2}=\mathrm{x}^{3}+\mathrm{t} \mathrm{x}^{2}+\mathrm{t}^{4} \mathrm{x}$

$-\Delta=t^{10}\left(t^{2}-1\right)=t^{10}(t+1)(t-1)$

4.2.18 Case 6A

$$
\begin{gathered}
\delta=9, \quad r=6 \quad \text { Type } \mathbf{I V}^{*} \\
y^{2}=x^{3}+t^{2} c_{0} x^{2}+t^{3} c_{1} x+t^{4} c_{2}, \quad t \nmid c_{2}, t \nmid c_{1} \\
-\Delta=t^{4} c_{0}^{2}\left(t^{6} c_{0} c_{2}-t^{6} d_{1}^{2}\right)+t^{9} c_{1}^{3}
\end{gathered}
$$

1. $\quad \mathbf{I V}^{*} 3 \quad \mathrm{y}^{2}=\mathrm{x}^{3}+\mathrm{t}^{2} \mathrm{x}^{2}+\mathrm{t}^{3} \mathrm{x}+\mathrm{t}^{4}\left(2 \mathrm{t}^{2}+1\right)$

$-\Delta=t^{12}-t^{9}=t^{9}(t-1)^{3}$

2. $\quad \mathbf{I V}^{*} \mathbf{2} \mathbf{1} \quad \mathrm{y}^{2}=\mathrm{x}^{3}+\mathrm{t}^{2} \mathrm{x}^{2}+2 \mathrm{t}^{3} \mathrm{x}+2 \mathrm{t}^{6}+\mathrm{t}^{5}+2 \mathrm{t}^{4}$

$-\Delta=t^{12}+2 t^{11}+2 t^{10}+t^{9}=t^{9}(t-1)^{2}(t+1)$

3. $\quad \mathbf{I V}^{*} \mathbf{1}^{3} \quad \mathrm{y}^{2}=\mathrm{x}^{3}+\mathrm{t}^{2} \mathrm{x}^{2}+\mathrm{t}^{4} \mathrm{x}+2 \mathrm{t}^{6}$

$-\Delta=t^{12}+t^{11}+2 t^{10}+t^{9}=t^{9}\left(t^{3}+t^{2}-t+1\right)$

4. $\quad \mathbf{I V}^{*} \mathbf{I I} \quad \mathrm{y}^{2}=\mathrm{x}^{3}+\mathrm{t}^{3}(2 \mathrm{t}+1) \mathrm{x}+\mathrm{t}^{4}$

$-\Delta=\mathrm{t}^{9}(2 \mathrm{t}+1)^{3}$

5. IV* III $y^{2}=x^{3}+t^{3}(t+2) x+t^{4}\left(t^{2}+t+1\right)$

$$
-\Delta=\mathrm{t}^{9}(\mathrm{t}+2)^{3}
$$




\subsubsection{Case 6B}

$$
\begin{gathered}
\delta=10, \quad r=6 \quad \text { Type } \mathbf{I V}^{*} \\
y^{2}=x^{3}+t^{2} c_{0} x^{2}+t^{4} d_{0} x+t^{4} c_{2} \quad t \nmid c_{2}, t \nmid c_{0} \\
\left.-\Delta=t^{4} c_{0}^{2}\left(t^{6} c_{0} c_{2}-t^{8} d_{0}^{2}\right)+t^{12} d_{0}^{3}\right)
\end{gathered}
$$

1. $\quad \mathbf{I V}^{*} \mathbf{1}^{2} \quad \mathrm{y}^{2}=\mathrm{x}^{3}+\mathrm{t} \mathrm{x}^{2}+\mathrm{t}^{4}\left(2 \mathrm{t}^{2}+1\right)$

$-\Delta=t^{12}+2 t^{10}=t^{10}(t+1)(t-1)$

2. $\quad \mathbf{I V}^{*} \mathbf{2} \quad \mathrm{y}^{2}=\mathrm{x}^{3}+\mathrm{t}^{2} \mathrm{c}_{0} \mathrm{x}^{2}+\mathrm{t}^{4}\left(\alpha \mathrm{t}^{2}+\alpha \mathrm{t}+\alpha\right)$ $-\Delta=\mathrm{t}^{10}\left(\mathrm{c}_{0}^{3} \alpha(\mathrm{t}-1)^{2}\right)$

4.2.20 Case 6C

$$
\begin{gathered}
\delta=12, \quad r=6 \quad \text { Type } \mathbf{I V}^{*} \\
y^{2}=x^{3}+t^{4} d_{0} x+t^{4} c_{2}, \quad t \nmid c_{2}, t \nmid d_{0} \\
-\Delta=t^{12} d_{0}^{3}
\end{gathered}
$$

\subsubsection{Case 7}

$$
\begin{gathered}
\delta=9, \quad r=7 \quad \text { Type III* } \\
y^{2}=x^{3}+t^{2} c_{0} x^{2}+t^{3} c_{1} x+t^{5} d_{1}, \quad t \nmid c_{1} \\
-\Delta=t^{4} c_{0}^{2}\left(t^{7} c_{0} d_{1}-t^{6} c_{1}^{2}\right)+t^{9} c_{1}^{3}
\end{gathered}
$$

1. III* 3 does not exist, $\mathrm{A}$

2. III $^{*} 21 \quad y^{2}=x^{3}+t^{2} x^{2}+t^{3}(t+1) x+t^{6}+t^{5}$

$-\Delta=t^{12}+2 t^{11}+2 t^{10}+t^{9}=t^{9}(t+1)\left(t^{2}+t+1\right)$

3. III* $^{*} 1^{3} \quad y^{2}=x^{3}+t^{2} x^{2}+t^{3}(t+1) x+2 t^{6}$

$-\Delta=2 t^{12}+t^{11}+2 t^{10}+t^{9}=t^{9}(2 t+1)\left(t^{2}+1\right)$

4. III* II $y^{2}=x^{3}+t^{3}(2 t+1) x+t^{5}(2+t)$

$-\Delta=\mathrm{t}^{9}(2 \mathrm{t}+1)^{3}$

5. III* III $y^{2}=x^{3}+t^{3}(2 t+1) x$

$-\Delta=\mathrm{t}^{9}(2 \mathrm{t}+1)^{3}$ 


\subsubsection{Case 8A}

$$
\begin{gathered}
\delta=11, \quad r=8 \quad \text { Type } \mathbf{I I}^{*} \\
\mathrm{y}^{2}=\mathrm{x}^{3}+\mathrm{t}^{2} \mathrm{c}_{0} \mathrm{x}^{2}+\mathrm{t}^{4} \mathrm{~d}_{0} \mathrm{x}+\mathrm{t}^{5} \mathrm{~d}_{1}, \quad \mathrm{t} \nmid \mathrm{d}_{1}, \mathrm{t} \nmid \mathrm{c}_{0} \\
-\Delta=\mathrm{t}^{4} \mathrm{c}_{0}^{2}\left(\mathrm{t}^{7} \mathrm{c}_{0} \mathrm{~d}_{1}-\mathrm{t}^{8} \mathrm{~d}_{0}^{2}\right)+\mathrm{t}^{12} \mathrm{~d}_{0}^{3}
\end{gathered}
$$

1. $\quad \mathbf{I I}_{2}^{*} \mathbf{1} \mathrm{y}^{2}=\mathrm{t}^{2} \mathrm{x}^{2}+\mathrm{t}^{4} \mathrm{x}+\mathrm{t}^{5}$

$-\Delta=t^{11}(t+1)$

4.2.23 Case 8B

$$
\begin{gathered}
\delta=12, \quad r=8 \quad \text { Type } \mathbf{I I}^{*} \\
\mathrm{y}^{2}=\mathrm{x}^{3}+\mathrm{t}^{4} \mathrm{~d}_{0} \mathrm{x}+\mathrm{t}^{5} \mathrm{~d}_{1}, \quad \mathrm{t} \nmid \mathrm{d}_{1}, \nmid \mathrm{d}_{0} \\
-\Delta=\mathrm{t}^{12} \mathrm{~d}_{0}^{3}
\end{gathered}
$$

\section{Conclusion and Summary of Results}

\subsection{General Results}

Of the 372 configurations checked above, having either multiplicative or additive singularities, 267 configurations were found to exist in characteristic three, and 105 were found not to exist. 227 configurations involving at least one additive singularity exist, while 68 involving at least one additive singularity did not. Of those configurations which are purely multiplicative, 40 exist, while 37 do not.

\subsection{Multiplicative Results in Comparison with other Char- acteristics}

As mentioned earlier, in different characteristics the additive singularities have such different properties that their configurations are not easily compared from characteristic to characteristic. However, this is not a problem with purely multiplicative fibres, so we compare them here.

There are 6 configurations that exist in characteristic zero, but not in characteristic three. But every configuration that exists in characteristic three also exists in characteristic zero. There are 6 configurations which exist in characteristic two but not characteristic three, and there are 10 configurations that exist in characteristic three but not characteristic two.

The the following table summarizes the results. A $\checkmark$ indicates that a rational elliptic surface with the indicated fibre distribution exists in the given case and characteristic, whereas an $X$ indicates that no such surface exists. 
Table 3: Existence of multiplicative fibre types

\begin{tabular}{|c|c|c|c|}
\hline Partition & Characteristic 0 & Characteristic 2 & Characteristic 3 \\
\hline $1^{12}$ & cl & cl & c \\
\hline $21^{10}$ & $\bar{\checkmark}$ & $\checkmark$ & $\checkmark$ \\
\hline $31^{9}$ & $\checkmark$ & $\checkmark$ & $\checkmark$ \\
\hline $2^{2} 1^{8}$ & $\bar{\checkmark}$ & $\checkmark$ & $\checkmark$ \\
\hline $41^{8}$ & $\checkmark$ & $\checkmark$ & $\checkmark$ \\
\hline $321^{7}$ & $\checkmark$ & $\checkmark$ & $\checkmark$ \\
\hline $51^{\prime}$ & $\checkmark$ & $\checkmark$ & $\checkmark$ \\
\hline $2^{3} 1^{6}$ & $\checkmark$ & $\checkmark$ & $\checkmark$ \\
\hline $421^{6}$ & $\checkmark$ & $\checkmark$ & $\checkmark$ \\
\hline $3^{2} 1^{6}$ & $\checkmark$ & $\checkmark$ & $\checkmark$ \\
\hline $61^{6}$ & $\checkmark$ & $\checkmark$ & $\checkmark$ \\
\hline $32^{2} 1^{5}$ & $\checkmark$ & $\checkmark$ & $\checkmark$ \\
\hline $521^{5}$ & $\checkmark$ & $\checkmark$ & $\checkmark$ \\
\hline $431^{5}$ & $\checkmark$ & $\checkmark$ & $\checkmark$ \\
\hline $71^{5}$ & $\checkmark$ & $\checkmark$ & $\checkmark$ \\
\hline $2^{4} 1^{4}$ & $\checkmark$ & $\checkmark$ & $\checkmark$ \\
\hline $42^{2} 1^{4}$ & $\checkmark$ & $\checkmark$ & $\checkmark$ \\
\hline $3^{2} 21^{4}$ & $\checkmark$ & $\checkmark$ & $\checkmark$ \\
\hline $621^{4}$ & $\checkmark$ & $\checkmark$ & $\checkmark$ \\
\hline $531^{4}$ & $\checkmark$ & $\checkmark$ & $\checkmark$ \\
\hline $4^{2} 1^{4}$ & $\checkmark$ & $\checkmark$ & $\checkmark$ \\
\hline $81^{4}$ & $\checkmark$ & $\checkmark$ & $\checkmark$ \\
\hline $32^{3} 1^{3}$ & $\checkmark$ & $\checkmark$ & $\checkmark$ \\
\hline $52^{2} 1^{3}$ & $\checkmark$ & $\checkmark$ & $\checkmark$ \\
\hline $4321^{3}$ & $\checkmark$ & $\checkmark$ & $\checkmark$ \\
\hline $721^{3}$ & $\checkmark$ & $\checkmark$ & $\checkmark$ \\
\hline $3^{3} 1^{3}$ & $\checkmark$ & $\checkmark$ & $X$ \\
\hline $631^{3}$ & $\checkmark$ & $\checkmark$ & $\bar{X}$ \\
\hline $541^{3}$ & $\bar{\checkmark}$ & $\checkmark$ & $\checkmark$ \\
\hline $91^{3}$ & $\checkmark$ & $\checkmark$ & $\bar{X}$ \\
\hline $2^{5} 1^{2}$ & $\checkmark$ & $X$ & $\checkmark$ \\
\hline $42^{3} 1^{2}$ & $\checkmark$ & $X$ & $\checkmark$ \\
\hline $3^{2} 2^{2} 1^{2}$ & $\checkmark$ & $\checkmark$ & $\checkmark$ \\
\hline $62^{2} 1^{2}$ & $\checkmark$ & $\bar{X}$ & $\checkmark$ \\
\hline $5321^{2}$ & $\checkmark$ & $\checkmark$ & $\checkmark$ \\
\hline $4^{2} 21^{2}$ & $\bar{\checkmark}$ & $X$ & $\checkmark$ \\
\hline $821^{2}$ & $\checkmark$ & $X$ & $\checkmark$ \\
\hline $43^{2} 1^{2}$ & $\bar{X}$ & $\bar{X}$ & $\bar{X}$ \\
\hline $731^{2}$ & $X$ & $X$ & $X$ \\
\hline $641^{2}$ & $X$ & $X$ & $X$ \\
\hline
\end{tabular}




\begin{tabular}{|c|c|c|c|}
\hline continued & om previous page & & \\
\hline Partition & Characteristic 0 & Characteristic 2 & Characteristic 3 \\
\hline $5^{2} 1^{2}$ & $\checkmark$ & $\checkmark$ & $\checkmark$ \\
\hline $101^{2}$ & $\bar{X}$ & $X$ & $\mathrm{X}$ \\
\hline $32^{4} 1$ & $\bar{\checkmark}$ & $\mathrm{X}$ & $\checkmark$ \\
\hline $52^{3} 1$ & $\bar{X}$ & $\bar{X}$ & $\bar{X}$ \\
\hline $432^{2} 1$ & $\checkmark$ & $X$ & $\checkmark$ \\
\hline $72^{2} 1$ & $\bar{X}$ & $\bar{X}$ & $\bar{X}$ \\
\hline $3^{3} 21$ & $\bar{\checkmark}$ & $\bar{\checkmark}$ & $\bar{X}$ \\
\hline 6321 & $\bar{\checkmark}$ & $\bar{X}$ & $\bar{X}$ \\
\hline 5421 & $\bar{X}$ & $X$ & $X$ \\
\hline 921 & $\bar{X}$ & $\bar{X}$ & $\bar{X}$ \\
\hline $53^{2} 1$ & $\bar{X}$ & $\bar{X}$ & $\bar{X}$ \\
\hline $4^{2} 31$ & $\bar{X}$ & $\bar{X}$ & $\bar{X}$ \\
\hline 831 & $\bar{X}$ & $\bar{X}$ & $X$ \\
\hline 741 & $\bar{X}$ & $\bar{X}$ & $\bar{X}$ \\
\hline 651 & $\bar{X}$ & $\bar{X}$ & $X$ \\
\hline 111 & $\bar{X}$ & $\bar{X}$ & $\bar{X}$ \\
\hline $2^{6}$ & $\checkmark$ & $\bar{X}$ & $\checkmark$ \\
\hline $42^{4}$ & $\bar{\checkmark}$ & $\bar{X}$ & $\bar{\checkmark}$ \\
\hline $3^{2} 2^{3}$ & $\bar{X}$ & $\bar{X}$ & $\bar{X}$ \\
\hline $62^{3}$ & $X$ & $X$ & $X$ \\
\hline $532^{2}$ & $X$ & $X$ & $X$ \\
\hline $4^{2} 2^{2}$ & $\bar{\checkmark}$ & $\bar{X}$ & $\checkmark$ \\
\hline $82^{2}$ & $\bar{X}$ & $X$ & $X$ \\
\hline $43^{2} 2$ & $\bar{X}$ & $\bar{X}$ & $\bar{X}$ \\
\hline 732 & $\bar{X}$ & $\bar{X}$ & $X$ \\
\hline 642 & $\bar{X}$ & $\bar{X}$ & $\bar{X}$ \\
\hline $5^{2} 2$ & $\bar{X}$ & $\bar{X}$ & $\bar{X}$ \\
\hline 102 & $\bar{X}$ & $\bar{X}$ & $\bar{X}$ \\
\hline $3^{4}$ & $\bar{\checkmark}$ & $\checkmark$ & $X$ \\
\hline $63^{2}$ & $\bar{X}$ & $\bar{X}$ & $\bar{X}$ \\
\hline 543 & $\bar{X}$ & $\bar{X}$ & $\bar{X}$ \\
\hline 93 & $X$ & $\bar{X}$ & $\bar{X}$ \\
\hline $4^{3}$ & $\bar{X}$ & $\bar{X}$ & $\bar{X}$ \\
\hline 84 & $\bar{X}$ & $\bar{X}$ & $X$ \\
\hline 75 & $\bar{X}$ & $\bar{X}$ & $\bar{X}$ \\
\hline $6^{2}$ & $\bar{X}$ & $\bar{X}$ & $\bar{X}$ \\
\hline 12 & $\bar{X}$ & $X$ & $X$ \\
\hline
\end{tabular}




\section{References}

[1] E. B. Dynkin, Semisimple subalgebras of semisimple Lie algebras, Mat. Sbornik N.S., Vol. 30(72) 1952, 349-462.

[2] W. E. Lang, Configurations of singular fibres on rational elliptic surfaces in characteristic two, Communications in Algebra, Vol. 28(12) 2000, 5813-5836.

[3] W. E. Lang, Extremal rational elliptic surfaces in characteristic p. II:Surfaces with three or fewer singular fibres, Arkiv for matematik, Vol. 32 1994, 423-438.

[4] R. Miranda, Persson's list of singular fibers for a rational elliptic surface, Mathematische Zeitschrift, Vol. 205 1990, 191-211.

[5] R. Miranda and U. Persson, On extremal rational elliptic surfaces, Mathematische Zeitschrift, Vol. 193 1986, 537-558.

[6] K. Oguiso and T. Shioda, The Mordell-Weil lattice of a rational elliptic surface, Comment. Math. Univ. St. Paul, Vol. 40(1) 1990, 83-99.

[7] U. Persson, Configurations of Kodaira fibers on rational elliptic surfaces, Mathematische Zeitschrift, Vol. 205(1) 1990, 1-47.

[8] T. Shioda, On the Mordell-Weil lattices, Comment. Math. Univ. St. Paul, Vol. 39(2) 1990, 211-240.

[9] J. H. Silverman, Advanced topics in the arithmetic of elliptic curves, Graduate Texts in Mathematics, Vol. 1511994. 